\title{
Les déterminants de la résilience et de la réussite scolaire : une approche bayésienne
}

Jérémie B. Dupuis

Université de Moncton

Jimmy Bourque

Collège royal des médecins et des chirurgiens du Canada

Salah-Eddine El Adlouni

Université de Moncton

\section{Résumé}

Avec un échantillon de 22113 élèves de la 7e à la 12e année du Nouveau-Brunswick, les déterminants de la résilience et de la réussite scolaire ont été identifiés à l'échelle provinciale et pour chacune des 7 zones de santé. Pour ce faire, cette étude adopte une approche méthodologique en trois étapes. D'abord, des régressions logistiques fréquentielles ont été utilisées pour faire un tri préliminaire des déterminants. Ensuite, des régressions logistiques

Canadian Journal of Education / Revue canadienne de l'éducation 44:4 (2021)

(C2021 Canadian Society for the Study of Education/ Société canadienne pour l'étude de l'éducation 
bayésiennes avec 20000 simulations MCMC ont permis de préciser les estimations et enfin, des calculs de probabilité à l'aide de profils d'élèves fictifs ont été effectués.

Mots-clés : résilience, réussite scolaire, déterminants de la santé, statistiques bayésiennes, Nouveau-Brunswick

\begin{abstract}
With a sample of 22,113 students from the 7th to the 12th grade in New Brunswick, the determinants of resilience and academic achievement were identified at the provincial level and for each of the province's seven health zones. To do so, this study used a threestep methodology. The first was two frequentist logistic regressions to do a preliminary sorting of the determinants. The second was a series of Bayesian logistic regressions with 20,000 MCMC simulations to narrow the estimations, and finally, probability calculations were run with fictitious student profiles.

Keywords: resilience, academic achievement, determinants of health, Bayesian statistics, New Brunswick
\end{abstract}




\section{Introduction}

Le plus récent Sondage sur le mieux-être des élèves du Nouveau-Brunswick (Conseil de la santé du Nouveau-Brunswick [CSNB], 2018) indique que 33,5\% des élèves de la 7e à la 12e année ont présenté des symptômes d'anxiété et 31,7 \% des symptômes de dépression au cours des 12 mois précédents. Bien que l'apparition de symptômes au début de l'adolescence ne mène pas toujours à un trouble anxieux ou dépressif diagnostiqué, il a été rapporté que $10,4 \%$ et $11,5 \%$ de la population du Nouveau-Brunswick âgée de 15 à 25 ans souffrent, respectivement, d'un trouble dépressif ou d'un trouble anxieux généralisé (Dezetter et al., 2016). Les données du Sondage sur le mieux-être des élèves du Nouveau-Brunswick (SMEENB) révèlent aussi qu'un élève sur quatre aurait une résilience considérée comme faible, ce qui signifie qu'il est incapable de s'adapter et de surmonter les difficultés (Luthar et Cicchetti, 2000).

Parallèlement, le rapport annuel de 2018-2019 du ministère de l'Éducation et du Développement de la petite enfance du Nouveau-Brunswick (MEDPE-NB, 2019a) indique que peu importe le système (francophone ou anglophone, le Nouveau-Brunswick étant officiellement bilingue), les rendements scolaires sont inquiétants. Chez les francophones, seulement $36 \%$ des élèves atteignent le niveau attendu en mathématiques de 10e année pour le parcours A (moins exigeant, minimum pour l'obtention du diplôme d'études secondaires) et $50 \%$ des élèves atteignent le niveau attendu en mathématiques de 10e année pour le parcours BC (plus exigeant, prépare aux études postsecondaires). Pour le cours de français de 11e année, seulement $21 \%$ des élèves atteignent le niveau attendu (MEDPE-NB, 2019b). Du côté anglophone, 62,3 \% des élèves de 10e année atteignent un niveau approprié en mathématiques et 78,1\% au cours d'anglais de 9e année (MEDPE-NB, 2020).

Pourquoi s'intéresser à la résilience et à la réussite scolaire des jeunes néobrunswickois ? En 2020, l'Institut canadien d'information sur la santé (ICIS) a constaté que le Nouveau-Brunswick se trouvait au troisième rang parmi les provinces avec le plus haut taux d'hospitalisation en raison de troubles mentaux pour les jeunes de 5 à 24 ans, derrière l'Île-du-Prince-Édouard et la Saskatchewan (ICIS, 2020). Une faible résilience chez les jeunes se traduit par une incapacité à identifier ses problèmes et à trouver l'aide appropriée (Ungar et al., 2013), ce qui se reflète par une diminution du bienêtre psychologique (Masten et al., 1999; Ungar et Theron, 2020) et limite le potentiel 
du jeune une fois rendu à l'âge adulte (Zolkoski et Bullock, 2012). Par ailleurs, le niveau de scolarité constitue un pilier et un prédicteur important du statut socioéconomique (American Psychological Association [APA], 2017). Selon cette même source, le statut socioéconomique est directement lié aux ressources familiales, aux aspirations de carrière des jeunes et au bienêtre psychologique de ceux-ci. Malheureusement, une faible réussite scolaire limite les capacités de l'individu à se défaire des effets associés au faible statut socioéconomique, qui devient alors un problème qui se perpétue de génération en génération (APA, 2017). L'éducation serait donc une manière de pallier les iniquités en matière de santé (Vable et al., 2018). En somme, s'intéresser à la résilience et à la réussite scolaire n'est pas seulement important pour améliorer la santé des jeunes au moment présent, mais aussi pour réduire les inégalités sociales des générations futures en permettant de cibler efficacement les programmes et les interventions en santé mentale et en prévention de l'échec scolaire.

L'objectif de cette étude est donc d'identifier les déterminants les plus importants de la résilience et de la réussite scolaire chez les jeunes de 12 à 18 ans du NouveauBrunswick. Pour ce faire, l'étude identifie l'effet des déterminants de la santé sur la résilience et la réussite scolaire. Elle compare la vulnérabilité des jeunes dans chacune des sept zones de santé du Nouveau-Brunswick tout en contrastant l'effet des divers déterminants d'une zone à l'autre à l'aide de profils de jeunes fictifs. Idéalement, les contrastes entre les zones de santé pourront servir à orienter de futures interventions.

\section{Cadre conceptuel}

Le CSNB a adopté, en 2014, un modèle épidémiologique de la santé visant à guider ses politiques et interventions : le modèle des déterminants de la santé (voir Figure 1). Ce modèle est inspiré de celui du Population Health Institute (PHI) de l'Université du Wisconsin (Booske et al., 2010). Dans ce modèle, les services de santé, les comportements liés à la santé, les facteurs socioéconomiques et les caractéristiques de l'environnement physique constituent les déterminants de la santé. Ces déterminants influent directement sur certains résultats de santé, en particulier sur la qualité et la durée de la vie d'un citoyen. Des pourcentages, obtenus à l'aide de perspectives historiques et de revues de la littérature (Booske et al., 2010), représentent le poids attribué à chaque 
catégorie et sont imputés aux catégories de déterminants. En raison de son lien avec le cadre d'analyse de la province et de son cadre de prestation de services, le modèle du CSNB a été utilisé comme cadre conceptuel général pour cet article et alimentera le choix des variables.

\section{Figure 1}

Le modèle des déterminants de la santé du Conseil de la santé du Nouveau-Brunswick

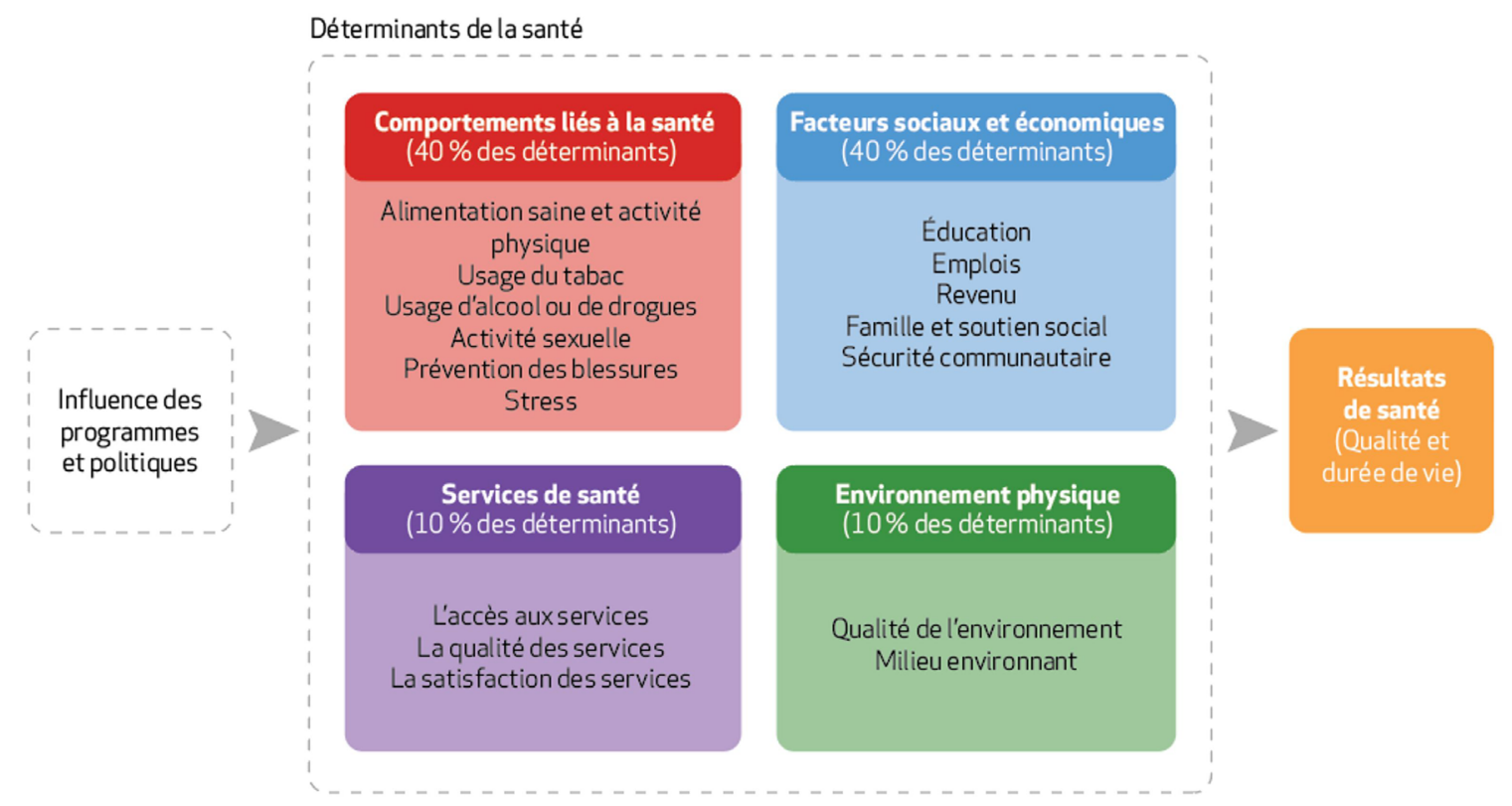

Note. Tiré de CSNB (2014).

\section{Méthodes}

\section{Devis et plan d'analyse}

Cette étude observationnelle déploie un devis corrélationnel rétrospectif. L'étude observe les relations entre les déterminants et les résultats de santé, sans en postuler la causalité. Afin de maximiser l'information pouvant être extraite des données, un plan d'analyse en trois étapes a été élaboré. Comme première étape, deux régressions 
logistiques fréquentielles - la première avec la résilience comme variable dépendante et la deuxième avec la réussite scolaire — ont été effectuées avec la version 26 du logiciel SPSS d'IBM (2019). Ces analyses ont permis d'effectuer un tri préliminaire des déterminants avant de procéder à la deuxième étape. La section intitulée « Variables indépendantes et analyses préliminaires » présentera les résultats de ces analyses. Comme deuxième étape, des régressions logistiques bayésiennes ont été effectuées avec la version R2019a du logiciel Matlab (2019). À cette étape, des régressions logistiques bayésiennes ont été réalisées à l'échelle des zones de santé et à l'échelle provinciale, et ce, séparément pour la résilience et la réussite scolaire, pour un total de 16 régressions logistiques bayésiennes. Les résultats pour chaque régression logistique bayésienne, donc les distributions à postériori, ont été obtenus à la suite de 20000 simulations générées avec la méthode de Monte-Carlo par Chaine de Markov (MCMC) avec l'algorithme de Metropolis-Hastings par échantillonnage de Gibbs (Robert, 2006). Les 10000 premières simulations générées de chaque régression ont été supprimées de l'estimation de la distribution à postériori afin d'assurer une " période de chauffe » suffisante pour obtenir une estimation approximant la stationnarité (Robert, 2006). La section intitulée « Survol des statistiques bayésiennes » présentera les avantages d'une telle approche. Finalement, comme dernière étape, les coefficients $\beta$ des distributions à postériori générés ont été utilisés afin de calculer les probabilités que des jeunes avec différents profils fictifs soient résilients ou atteignent les standards scolaires. Ces probabilités, calculées manuellement à l'aide de l'Équation 1, sont présentées pour chaque zone de santé et pour la province. Dans cette équation, $X$ indique si le déterminant est présent ( 0 pour non, 1 pour oui), $\beta$ est le coefficient de régression logistique bayésienne et $i$ représente le nombre de déterminants. Enfin, pour mieux illustrer les différences entre les zones, la probabilité a été recalculée après l'addition de chaque déterminant de la santé du profil fictif.

$$
p=\prod_{i=1}^{n} \frac{e^{X_{i} \beta_{i}+X_{n} \beta_{n}}}{1+e^{X_{i} \beta_{i}+X_{n} \beta_{n}}}
$$

\section{Données}

Le SMEENB du CSNB est une enquête transversale qui vise à examiner les perceptions, les attitudes et les comportements des élèves dans un certain nombre de domaines liés à leur mieux-être (CSNB, 2018). L'enquête a été menée en collaboration avec le MEDPE-NB 
auprès de 69,6\% de la population totale des élèves de la 7e à la 12e année de la province au cours de l'année scolaire 2015-2016 (32 677 des 46939 élèves possibles). Il est à noter que le SMEENB a aussi été utilisé auprès des élèves de la 6e année de la province, mais puisque ces élèves répondaient à une série de questions différente de celle destinée aux élèves de la 7e à la 12e année, ils n'ont pas été inclus dans cette étude. À la suite des analyses préliminaires sur les données du SMEENB, l'échantillon de cette étude comprend 22113 élèves. Ces données peuvent être analysées tant au niveau provincial qu'au niveau des sept zones de santé. Ces zones ont été déterminées de manière législative (CSNB, 2021) : Moncton/Sud-est (zone 1), Fundy/Saint John (zone 2), Fredericton/La vallée (zone 3), Madawaska/Nord-Ouest (zone 4), Restigouche (zone 5), Bathurst/Péninsule acadienne (zone 6) et Miramichi (zone 7). Plusieurs variables démographiques sont incluses dans le SMEENB; il sera donc possible de considérer leurs effets sur la résilience et la réussite scolaire. Le Tableau 1 décrit l'échantillon provincial par zone de santé.

\section{Tableau 1}

Tableau descriptif de l'échantillon provincial et de chaque zone de santé

\begin{tabular}{|c|c|c|c|c|c|c|c|c|}
\hline & $\begin{array}{c}\text { NB } \\
n=22113\end{array}$ & $\begin{array}{c}\text { Zone } 1 \\
\text { Moncton } \\
n=6533 \\
\end{array}$ & $\begin{array}{c}\text { Zone } 2 \\
\text { Saint-Jean } \\
n=5893 \\
\end{array}$ & $\begin{array}{c}\text { Zone } 3 \\
\text { Fredericton } \\
n=4452\end{array}$ & $\begin{array}{c}\text { Zone } 4 \\
\text { Madawaska } \\
n=1547\end{array}$ & $\begin{array}{c}\text { Zone } 5 \\
\text { Restigouche } \\
n=605\end{array}$ & $\begin{array}{c}\text { Zone } 6 \\
\text { Bathurst } \\
n=1780 \\
\end{array}$ & $\begin{array}{c}\text { Zone } 7 \\
\text { Miramichi } \\
n=1303\end{array}$ \\
\hline Francophones & $\begin{array}{c}6507 \\
(29,4 \%)\end{array}$ & $\begin{array}{c}2654 \\
(40,6 \%)\end{array}$ & $\begin{array}{c}148 \\
(2,5 \%)\end{array}$ & $\begin{array}{c}412 \\
(9,3 \%)\end{array}$ & $\begin{array}{c}1348 \\
(87,1 \%)\end{array}$ & $\begin{array}{c}387 \\
(64,0 \%)\end{array}$ & $\begin{array}{c}1334 \\
(74,9 \%)\end{array}$ & $\begin{array}{c}224 \\
(17,2 \%)\end{array}$ \\
\hline Anglophones & $\begin{array}{c}15606 \\
(70,6 \%)\end{array}$ & $\begin{array}{c}3879 \\
(59,4 \%)\end{array}$ & $\begin{array}{c}5745 \\
(97,5 \%)\end{array}$ & $\begin{array}{c}4040 \\
(90,7 \%)\end{array}$ & $\begin{array}{c}199 \\
(12,9 \%)\end{array}$ & $\begin{array}{c}218 \\
(36,0 \%)\end{array}$ & $\begin{array}{c}446 \\
(25,1 \%)\end{array}$ & $\begin{array}{c}1079 \\
(82,8 \%)\end{array}$ \\
\hline Garçons & $\begin{array}{c}10180 \\
(46,0 \%)\end{array}$ & $\begin{array}{c}3046 \\
(46,6 \%)\end{array}$ & $\begin{array}{c}2705 \\
(45,9 \%)\end{array}$ & $\begin{array}{c}2104 \\
(47,3 \%)\end{array}$ & $\begin{array}{c}663 \\
(42,9 \%)\end{array}$ & $\begin{array}{c}263 \\
(43,5 \%)\end{array}$ & $\begin{array}{c}823 \\
(46,2 \%)\end{array}$ & $\begin{array}{c}576 \\
(44,4 \%)\end{array}$ \\
\hline Filles & $\begin{array}{c}11658 \\
(52,7 \%)\end{array}$ & $\begin{array}{c}3414 \\
(52,3 \%)\end{array}$ & $\begin{array}{c}3109 \\
(52,8 \%)\end{array}$ & $\begin{array}{c}2283 \\
(51,3 \%)\end{array}$ & $\begin{array}{c}872 \\
(56,4 \%)\end{array}$ & $\begin{array}{c}329 \\
(54,4 \%)\end{array}$ & $\begin{array}{c}942 \\
(52,9 \%)\end{array}$ & $\begin{array}{c}709 \\
(54,4 \%)\end{array}$ \\
\hline Sexe non-binaire & $\begin{array}{c}275 \\
(1,2 \%)\end{array}$ & $\begin{array}{c}73 \\
(1,1 \%)\end{array}$ & $\begin{array}{c}79 \\
(1,3 \%)\end{array}$ & $\begin{array}{c}65 \\
(1,5 \%)\end{array}$ & $\begin{array}{c}12 \\
(0,8 \%)\end{array}$ & $\begin{array}{c}13 \\
(2,1 \%)\end{array}$ & $\begin{array}{c}15 \\
(0,8 \%)\end{array}$ & $\begin{array}{c}18 \\
(1,4 \%)\end{array}$ \\
\hline LGBTQ & $\begin{array}{c}3098 \\
(14,0 \%)\end{array}$ & $\begin{array}{c}880 \\
(13,5 \%)\end{array}$ & $\begin{array}{c}867 \\
(14,7 \%)\end{array}$ & $\begin{array}{c}709 \\
(15,9 \%)\end{array}$ & $\begin{array}{c}204 \\
(13,2 \%)\end{array}$ & $\begin{array}{c}82 \\
(13,6 \%)\end{array}$ & $\begin{array}{c}215 \\
(12,1 \%)\end{array}$ & $\begin{array}{c}141 \\
(10,8 \%)\end{array}$ \\
\hline Autochtones & $\begin{array}{c}1150 \\
(5,2 \%)\end{array}$ & $\begin{array}{c}324 \\
(5,0 \%)\end{array}$ & $\begin{array}{c}224 \\
(3,8 \%)\end{array}$ & $\begin{array}{c}313 \\
(7,0 \%)\end{array}$ & $\begin{array}{c}44 \\
(2,8 \%)\end{array}$ & $\begin{array}{c}61 \\
(10,1 \%)\end{array}$ & $\begin{array}{c}55 \\
(3,1 \%)\end{array}$ & $\begin{array}{c}129 \\
(9,9 \%)\end{array}$ \\
\hline Immigrants & $\begin{array}{c}1128 \\
(5,1 \%)\end{array}$ & $\begin{array}{c}375 \\
(5,7 \%)\end{array}$ & $\begin{array}{c}332 \\
(5,6 \%)\end{array}$ & $\begin{array}{c}310 \\
(7,0 \%)\end{array}$ & $\begin{array}{c}35 \\
(2,3 \%)\end{array}$ & $\begin{array}{c}8 \\
(1,3 \%)\end{array}$ & $\begin{array}{c}40 \\
(2,2 \%)\end{array}$ & $\begin{array}{c}28 \\
(2,1 \%)\end{array}$ \\
\hline $\begin{array}{l}\text { Besoins } \\
\text { d'apprentissage }\end{array}$ & $\begin{array}{c}3803 \\
(17,2 \%)\end{array}$ & $\begin{array}{c}1149 \\
(17,6 \%)\end{array}$ & $\begin{array}{c}1037 \\
(17,6 \%)\end{array}$ & $\begin{array}{c}740 \\
(16,6 \%)\end{array}$ & $\begin{array}{c}202 \\
(13,1 \%)\end{array}$ & $\begin{array}{c}143 \\
(23,6 \%)\end{array}$ & $\begin{array}{c}356 \\
(20,0 \%)\end{array}$ & $\begin{array}{c}176 \\
(13,5 \%)\end{array}$ \\
\hline Âge moyen & 14,7 & 14,6 & 14,7 & 14,7 & 14,8 & 14,5 & 14,4 & 15,0 \\
\hline
\end{tabular}

Variables dépendantes. Les variables dépendantes utilisées proviennent du SMEENB. Douze items du SMEENB ont été utilisés pour évaluer la résilience. Les 
élèves devaient indiquer si des affirmations telles que « Je suis capable de résoudre mes problèmes sans me nuire ou nuire aux autres » ou « J'essaie de terminer ce que je commence » leur correspondaient, soit $1=P a s d u$ tout, $2=U n$ peu, $3=$ Moyennement, 4 = Beaucoup ou 5 = Énormément . Les scores à ces 12 items ont ensuite été additionnés, puis, si la somme atteignait 45 ou plus, leur résilience était considérée comme modérée ou élevée. Le seuil de coupure établi à 45 a été déterminé à l'aide d'une régression prédisant l'absence ou la présence de comportements négatifs, comme la consommation de drogues et les comportements oppositionnels (CSNB, 2015). Utilisant le lambda-6 de Guttman, le meilleur estimateur de la consistance interne disponible sur SPSS (Bourque et al., 2019), les 12 items de la résilience démontrent une consistance interne de .91, ce qui est bien au-delà du seuil recommandé de .80 (Bourque et al., 2019).

Pour la réussite scolaire, le SMEENB demande aux élèves « Parmi les réponses suivantes, laquelle décrit le mieux tes résultats scolaires au cours de la dernière année ?». Les choix de réponses étaient « Excellents (surtout des A/90\% ou plus) 》, " Supérieurs à la moyenne (surtout des A et des B/de 80 à $89 \%$ ) 》, «Moyens (surtout des B et des C/de 70 à $79 \%$ ) 》, "Inférieurs à la moyenne (surtout des C/de 60 à $69 \%$ ) 》 et «Faible (surtout des notes inférieures C/59\% ou moins) ». Afin de mieux identifier les déterminants du niveau attendu de réussite scolaire ( $70 \%$ ou plus) de la province du Nouveau-Brunswick (MEDPE-NB, 2019b), la réussite a été dichotomisée de sorte à attribuer aux élèves avec $90 \%$ ou plus, 80 à $89 \%$ et 70 à $79 \%$ de moyenne une valeur de 1 , et ceux avec 60 à $69 \%$ ou $59 \%$ ou moins une valeur de 0 .

Variables indépendantes et analyses préliminaires. Le Tableau 2 présente les variables indépendantes (déterminants de la santé). Ces variables ont été choisies selon les associations trouvées dans la littérature entre chaque déterminant et les variables résultantes, ainsi que selon la disponibilité des données du SMEENB. Elles s'inspirent également du modèle des déterminants de la santé du CSNB (2014), mais certaines variables de ce modèle, comme l'activité sexuelle et le stress, ont été omises de l'étude puisqu'elles n'étaient pas consignées dans le SMEENB. Toutes ces variables sont dichotomiques, donc une valeur de 1 indique la présence du déterminant, et une valeur de 0 indique son absence. Le Tableau 2 contient aussi des rapports de cotes et des intervalles de confiance de $95 \%$ obtenus à l'aide de régressions logistiques fréquentielles. Lorsque le déterminant a un effet protecteur (favorise la résilience ou la 
réussite), le rapport de cote et son intervalle de confiance seront supérieurs à 1.00, alors que s'ils sont entre 0.00 et 1.00 , l'effet est néfaste. Si l'intervalle de confiance contient la valeur 1.00, le déterminant n'a pas d'effet significatif. Les résultats des régressions logistiques fréquentielles présentés au Tableau 2 ont permis de faire un tri préliminaire des déterminants avant les analyses principales de l'étude.

\section{Tableau 2}

Variables indépendantes de l'étude, leur rôle et les résultats des régressions logistiques fréquentielles préliminaires

\begin{tabular}{llcc}
\hline Déterminant & Rôle & Résilience & Réussite \\
\hline Langue & Variable démographique, & 1,06 & $0,45^{*}$ \\
& association à déterminer & {$[0,97,1,16]$} & {$[0,40,0,51]$} \\
Sexe & Variable démographique, & $0,75^{*}$ & 0,80 \\
& association à déterminer & {$[0,69,0,82]$} & {$[0,53,1,12]$} \\
Statut LGBTQ & Variable démographique, & $0,55^{*}$ & 1,00 \\
& facteur de vulnérabilité & {$[0,50,0,62]$} & {$[0,85,1,18]$} \\
Statut immigrant & Variable démographique, & 1,00 & $1,39^{*}$ \\
& facteur de vulnérabilité & {$[0,83,1,20]$} & {$[1,01,1,94]$} \\
Besoins d'apprentissage particuliers & Variable démographique, & $0,68^{*}$ & $0,42^{*}$ \\
& facteur de vulnérabilité & {$[0,61,0,75]$} & {$[0,37,0,48]$} \\
Environnement bâti et naturel & Variable mesurée, facteur & $1,55^{*}$ & $0,82^{*}$ \\
& de protection & {$[1,43,1,69]$} & {$[0,72,0,93]$} \\
Besoins psychologiques famille & Variable mesurée, facteur & $5,15^{*}$ & 1,18 \\
& de protection & {$[4,71,5,63]$} & {$[1,00,1,36]$} \\
Besoins psychologiques amis & Variable mesurée, facteur & $3,27^{*}$ & 1,08 \\
& de protection & {$[2,98,3,61]$} & {$[0,94,1,25]$} \\
Besoins psychologiques école & Variable mesurée, facteur & $3,76^{*}$ & $3,27^{*}$ \\
& de protection & {$[3,46,4,08]$} & {$[2,86,3,75]$} \\
Sécurité communautaire & Variable mesurée, facteur & $2,04^{*}$ & $1,31^{*}$ \\
& de protection & {$[1,88,2,21]$} & {$[1,16,1,49]$} \\
Insécurité alimentaire & Variable mesurée, facteur & $0,55^{*}$ & $0,61^{*}$ \\
& de vulnérabilité & {$[0,46,0,66]$} & {$[0,50,0,74]$} \\
Exercice & Variable mesurée, facteur & $1,42^{*}$ & $1,35^{*}$ \\
& de protection & {$[1,28,1,58]$} & {$[1,16,1,57]$} \\
Tabac quotidien ou occasionnel & Variable mesurée, facteur & $0,60^{*}$ & $0,47^{*}$ \\
& de vulnérabilité & {$[0,52,0,69]$} & {$[0,40,0,56]$} \\
Alcool mensuel & Variable mesurée, facteur & 0,94 & $0,83^{*}$ \\
& de vulnérabilité & {$[0,82,1,08]$} & {$[0,70,0,96]$} \\
Risques de blessures & Variable mesurée, facteur & 0,82 & $0,79^{*}$ \\
& de vulnérabilité & {$[0,65,1,03]$} & {$[0,65,0,97]$} \\
\hline
\end{tabular}

Note. $*$ Indique un rapport de cote statistiquement significatif $(p<.05)$ 
Pour évaluer les déterminants de la résilience et de la réussite scolaire à l'aide de régressions logistiques fréquentielles, il est recommandé d'avoir au moins 10 fois plus de participants dans la catégorie de la variable dépendante qui en compte le moins que le nombre total de variables, ici, 190 participants (Bourque et El Adlouni, 2016). La modalité la moins fréquente des variables dépendantes comptant 283 participants, ce postulat est respecté. Après la simple élimination des cas pour lesquels il manquait au moins une donnée, $67,7 \%$ des participants ont été inclus dans l'analyse. Souvent, une imputation multiple est recommandée lorsqu'une proportion importante de l'échantillon est exclue des analyses (Little et Rubin, 2002). Cependant, les calculs de puissance réalisés avec la version 3.1 du logiciel G*Power (Faul et al., 2009) indiquent que même avec $68,4 \%$ de l'échantillon total et une petite taille d'effet attendue, la puissance obtenue approxime la valeur de 1 . Il est aussi nécessaire de vérifier si un mécanisme sous-jacent pourrait expliquer les données manquantes. En d'autres termes, il s'agit de vérifier si les participants inclus dans l'analyse diffèrent significativement des participants en étant exclus. Pour ce faire, une variable dichotomique a été créée pour laquelle les participants recevaient un score de 1 s'ils étaient inclus dans l'analyse et un score de 0 s'ils en étaient exclus. Cette variable a ensuite été croisée avec chaque déterminant afin d'évaluer la taille de l'effet en utilisant phi $(\varphi)$ comme estimateur. La taille de l'effet la plus importante s'observait entre le sexe et la résilience, où $\varphi=.09$, ce qui représente une petite taille de l'effet selon Cohen (1988). Par conséquent, il a été considéré raisonnable de supposer que le mécanisme des données manquantes était aléatoire, indiquant que la probabilité qu'une variable soit manquante ne dépend pas de la variable dépendante, mais peut être liée à d'autres variables comprises dans la base de données (Bourque et El Adlouni, 2016). Puisqu'une distribution aléatoire des données manquantes n'est pas problématique, les données manquantes seront traitées par simple élimination. Comme aucun participant n'avait une distance de Cook supérieure à 1, l'ensemble de données ne contenait pas de données déviantes multivariées. La colinéarité a été évaluée à l'aide de valeurs de tolérance, de facteurs d'inflation de la variance (VIF) et d'indices de condition. Les valeurs de tolérance étaient toutes supérieures au seuil acceptable de .10, aucune colinéarité n'est donc constatée (Bourque et El Adlouni, 2016).

Survol des statistiques bayésiennes. En statistique inférentielle, il y a deux approches principales : bayésienne et fréquentielle. Dans l'approche fréquentielle, les 
estimations des paramètres du modèle, comme la probabilité d'occurrence, reposent uniquement sur les résultats d'un échantillon aléatoire de la population. Cet énoncé de probabilité est ensuite converti en une inférence sur la vraisemblance du paramètre par rapport au comportement moyen du modèle pour tous les échantillons possibles (Bolstad et Curran, 2016). Cependant, l'approche fréquentielle ne tient compte d'aucune connaissance préalable du paramètre. En termes simples, on tente de calculer la probabilité des estimateurs obtenus d'un échantillon en supposant la véracité de l'hypothèse nulle, quelle que soit sa plausibilité réelle pour la population échantillonnée.

L'alternative à la méthode fréquentielle est l'approche bayésienne. Elle consiste à inclure toutes les informations disponibles avant d'analyser l'échantillon pour l'estimation des paramètres. Comme pour l'approche fréquentielle, les paramètres de la population sont inconnus, mais au lieu de les fixer, l'approche bayésienne considère différentes sources d'information, soit historiques, régionales ou d'autres expériences antérieures similaires, afin de les combiner aux informations fournies par les données (Bolstad et Curran, 2016). L'information antérieure et les données sont combinées à l'aide du théorème de Bayes, présenté à l'Équation 2, qui repose sur les principes de la probabilité conditionnelle. Brièvement, une probabilité conditionnelle réfère à la probabilité qu'un évènement $B$ se produise, sachant qu'un autre évènement, dénoté $A$, s'est produit. Ensuite, la distribution à postériori (la meilleure estimation de la distribution d'intérêt compte tenu des connaissances antérieures et des données de l'échantillon) dépend à la fois de la distribution antérieure (la meilleure estimation de la distribution basée uniquement sur les connaissances disponibles avant l'analyse des données de l'échantillon) et des données observées par la fonction de vraisemblance, minimisant la distance entre l'estimation à postériori et la vraie distribution (qu'on ne peut pas mesurer). Conséquemment, les estimations de probabilités à postériori incluent dans le processus d'estimation non seulement les données de l'échantillon, mais aussi toute l'information disponible avant d'observer l'échantillon, mettant en valeur l'avantage principal de l'approche bayésienne.

$$
P(B \mid A)=\frac{P(A \mid B) \cdot P(B)}{P(A \cap B)+P(A \cap \bar{B})}
$$


Distributions à priori. Une étape importante des analyses bayésiennes est le choix des distributions à priori. Les à priori sont des hypothèses du modèle et, comme la plupart des hypothèses, peuvent varier dans leur capacité à être informatives. En conséquence, ce qui rend les à priori utiles, c'est leur maniabilité : ils peuvent être étendus, révisés ou rejetés en fonction de leur pertinence par rapport aux données étudiées. Par exemple, supposons que chaque valeur d'une distribution de -1 à 1 est possible, alors la distribution à priori sera simplement une fonction de densité de probabilité uniforme allant de -1 à 1 . Cependant, si les connaissances antérieures sur le sujet voient généralement un résultat positif, la distribution à priori peut être modifiée pour une étude subséquente afin de tenir compte des connaissances actuelles, entrainant une distribution à priori révisée avec une plage de 0 à 1 . En choisissant des distributions à priori appropriées, les chercheurs peuvent intégrer à leurs analyses des connaissances utiles et des contraintes significatives (Vanpaemel et Lee, 2012; Wagenmakers et al., 2018).

Van de Schoot et al. (2014) suggèrent qu'il existe deux scénarios possibles. Dans le premier, les chercheurs n'ont pas suffisamment d'informations préalables pour établir une distribution à priori précise : on choisira alors une distribution peu informative, la distribution uniforme par exemple. Dans le deuxième scénario, les chercheurs disposent d'une information préalable plus ou moins considérable sur la distribution des paramètres et leur exactitude : il est alors possible d'utiliser cette distribution informative, d'autant plus que l'information à son égard est abondante et cohérente, comme à priori.

Dans le cadre de cette étude, des articles présentant des rapports de cotes, des intervalles de crédibilité et des étendues de valeurs probables, ont été recherchés afin d'établir les distributions à priori (la note du Tableau 3 liste les articles utilisés). Lorsque les articles ne donnaient pas de rapports de cotes spécifiques, d'intervalles de crédibilité ou de données pour calculer les rapports de cotes, mais indiquaient une forte association entre les deux variables, nous avons assigné des intervalles de crédibilité plus polarisés. Dans les cas où l'association entre un déterminant de la santé et un résultat était forte, et où le déterminant agissait comme facteur de protection, un intervalle de crédibilité de $[.40, .80]$ a été attribué (Cady, 2014; Roustit et al., 2010; Wang et Eccles, 2013; Wight et al., 2006). Ces limites d'intervalle ont été choisies après avoir observé que peu d'articles répertoriés présentaient des rapports de cotes inférieurs à .40 ou supérieurs à 3.00. Lorsque l'association entre un déterminant de la santé et un résultat était plus faible, des intervalles de crédibilité de $[.80,1.40]$ ont été attribués aux déterminants de la vulnérabilité et $[.60,1.20]$ aux 
déterminants de la protection (Herman et al., 2015; Sektnan et al., 2010). Il est à noter que ces intervalles de crédibilité indiquent une direction, mais laissent place à un certain degré d'incertitude, car ils contiennent 1.00, qui indique l'absence d'effet significatif.

Pour les simulations avec les antécédents (à priori) susmentionnés, les moyennes sont centrées entre les limites de l'intervalle et suivent une fonction de probabilité normale, rendant les simulations avec des moyennes plus proches des limites de l'intervalle moins fréquentes que les moyennes centrées. Enfin, lorsque très peu d'études ont pu être trouvées ou lorsque les résultats des associations entre les déterminants et les variables des résultats étaient incohérents, une distribution de la densité de probabilité uniforme a été attribuée, ce qui signifie que toutes les valeurs de la distribution sont équiprobables. Afin d'alléger les simulations et l'interprétation des résultats, seuls les déterminants de la santé identifiés comme statistiquement significatifs à la suite des régressions logistiques fréquentielles préliminaires sont utilisés dans les simulations bayésiennes. Le Tableau 3 énumère les $a ̀$ priori établis pour les paramètres des variables indépendantes.

\section{Tableau 3}

Distributions des à priori

\begin{tabular}{lcc}
\hline Déterminant & Résilience & Réussite scolaire \\
\hline Langue - Français & -- & non-informative \\
Sexe (masculin) & faible $[0,80,1,40]$ & -- \\
Sexe (non-binaire) & -- & non-informative \\
Statut LGBTQ & élevée $[0,40,0,80]$ & -- \\
Statut immigrant & -- & non-informative \\
Besoins d'apprentissage particuliers & faible $[0,80,1,40]$ & élevée $[0,40,0,80]$ \\
Environnement bâti et naturel & faible $[0,80,1,40]$ & faible $[0,80,1,40]$ \\
Besoins psychologiques famille & élevée $[1,20,3,00]$ & -- \\
Besoins psychologiques amis & élevée $[1,20,3,00]$ & -- \\
Besoins psychologiques école & élevée $[1,20,3,00]$ & élevée $[1,20,3,00]$ \\
Sécurité communautaire & faible $[0,80,1,40]$ & faible $[0,80,1,40]$ \\
Insécurité alimentaire & élevée $[0,40,0,80]$ & élevée $[0,40,0,80]$ \\
Exercice & faible $[0,80,1,40]$ & faible $[0,80,1,40]$ \\
Tabac quotidien ou occasionnel & $0,70[0,50,1,00]$ & élevée $[0,40,0,80]$ \\
Alcool mensuel & -- & élevée $[0,40,0,80]$ \\
Risques de blessures & -- & élevée $[0,40,0,80]$ \\
\hline
\end{tabular}

Note. Parmi les articles utilisés pour établir les distributions à priori se retrouvent : Balsa et al. (2011), Barry (2009), Berman et al. (2008), Borofsky et al. (2013), Cady (2014), Daley et Birchwood (2010), Deci et Ryan (2000), Donnelly et al. (2016), Forrest et al. (2011), Hair et al. (2009), Halpern (2014), Herman et al. (2015), King (2015), Melchior et al. (2012), Milam et al. (2010), Reading et Wien (2009), Roustit et al. (2010), Russell et Fish (2016), Wang et Eccles (2013), Wang et Holcombe (2010), Zolkoski et Bullock (2012). 


\section{Résultats des régressions logistiques bayésiennes}

\section{Résilience}

Le Tableau 4 présente les résultats des régressions logistiques bayésiennes pour la résilience. Au niveau provincial, trois déterminants surpassent les autres dans leur capacité de promouvoir la résilience chez les jeunes : les besoins psychologiques comblés par la famille, les amis et à l'école. Les besoins psychologiques satisfaits par les membres de la famille sont particulièrement importants, les élèves satisfaits étant 5,11 fois (I.C. 95 $\%=[4.77,5.57])$ plus susceptibles d'avoir une résilience moyenne ou élevée. Cependant, les deux autres types de soutien sont aussi très importants, car les élèves dont les besoins psychologiques sont comblés par leurs amis sont 3,17 fois (I.C. $95 \%=[2.91,3.38]$ ) plus susceptibles d'être résilients, et les élèves dont les besoins psychologiques sont satisfaits à l'école sont 3,85 fois (I.C. $95 \%=[3.58,4.18]$ ) plus susceptibles de l'être. D'autres facteurs de protection dignes de mention comprennent l'environnement bâti et naturel entourant l'élève, la sécurité communautaire et l'exercice. En ce qui concerne les facteurs de vulnérabilité au niveau provincial, le tabagisme quotidien ou occasionnel est le facteur prédictif le plus important, les élèves fumeurs n'étant que 0,53 fois (I.C. $95 \%=[.49$, .57]) aussi susceptibles d'être résilients que leurs homologues non-fumeurs. Enfin, les élèves LGBTQ et ceux qui vivent dans l'insécurité alimentaire ne sont que 0,57 fois (I.C. $95 \%=[.54, .61])$ aussi susceptibles d'être résilients que leurs pairs. Il est intéressant de noter que la consommation de cannabis n'est pas un indicateur significatif de la résilience chez les jeunes. 


\section{Tableau 4}

Rapports de cotes, intervalles de crédibilité à $95 \%$ et bêtas pour la résilience

\begin{tabular}{|c|c|c|c|c|c|c|c|c|}
\hline \multirow[b]{2}{*}{ Variable-sous-groupe } & \multicolumn{8}{|c|}{ Zone } \\
\hline & NB & 1 & 2 & 3 & 4 & 5 & 6 & 7 \\
\hline \multicolumn{9}{|l|}{ Rapports de cotes } \\
\hline Males & $\begin{array}{c}\mathbf{0 , 8 1} * \\
{[0,77,} \\
0,85]\end{array}$ & $\begin{array}{c}0,81 * \\
{[0,74,} \\
0,91]\end{array}$ & $\begin{array}{c}0,81 * \\
{[0,74,} \\
0,93]\end{array}$ & $\begin{array}{c}0,86^{*} \\
{[0,76,} \\
0,97]\end{array}$ & $\begin{array}{c}0,65 * \\
{[0,56} \\
0,89]\end{array}$ & $\begin{array}{l}0,71 \\
{[0,58} \\
1,01]\end{array}$ & $\begin{array}{c}0,68 * \\
{[0,59,} \\
0,83]\end{array}$ & $\begin{array}{c}0,80 * \\
{[0,64,} \\
0,98]\end{array}$ \\
\hline Statut LGBTQ & $\begin{array}{c}0,57 * \\
{[0,54} \\
0,61]\end{array}$ & $\begin{array}{c}0,55 * \\
{[0,49} \\
0,63]\end{array}$ & $\begin{array}{c}0,54 * \\
{[0,47} \\
0,62]\end{array}$ & $\begin{array}{c}0,60 * \\
{[0,52,} \\
0,69]\end{array}$ & $\begin{array}{c}0,64 * \\
{[0,51} \\
0,82]\end{array}$ & $\begin{array}{c}0,60 * \\
{[0,43} \\
0,88]\end{array}$ & $\begin{array}{c}0,50 * \\
{[0,42,} \\
0,65]\end{array}$ & $\begin{array}{c}0,75^{*} \\
{[0,61} \\
0,94]\end{array}$ \\
\hline Besoins d'apprentissage & $\begin{array}{c}0,76^{*} \\
{[0,71} \\
0,82]\end{array}$ & $\begin{array}{c}0,78 * \\
{[0,66,} \\
0,88]\end{array}$ & $\begin{array}{c}0,77 * \\
{[0,66} \\
0,88]\end{array}$ & $\begin{array}{c}0,70 * \\
{[0,62,} \\
0,88]\end{array}$ & $\begin{array}{c}0,75^{*} \\
{[0,59} \\
0,98]\end{array}$ & $\begin{array}{l}0,86 \\
{[0,62,} \\
1,26]\end{array}$ & $\begin{array}{c}0,87 \\
{[0,69} \\
1,05]\end{array}$ & $\begin{array}{c}0,71 * \\
{[0,51} \\
0,96]\end{array}$ \\
\hline Environnement bâti et naturel & $\begin{array}{c}1,52 * \\
{[1,45,} \\
1,61]\end{array}$ & $\begin{array}{c}1,54^{*} \\
{[1,38,} \\
1,77]\end{array}$ & $\begin{array}{c}1,66^{*} \\
{[1,48} \\
1,91]\end{array}$ & $\begin{array}{c}1,48^{*} \\
{[1,31} \\
1,68]\end{array}$ & $\begin{array}{c}1,32 * \\
{[1,08} \\
1,72]\end{array}$ & $\begin{array}{c}1,25^{*} \\
{[1,01,} \\
1,98]\end{array}$ & $\begin{array}{c}1,33^{*} \\
{[1,15,} \\
1,58]\end{array}$ & $\begin{array}{c}1,22 * \\
{[1,01,} \\
1,48]\end{array}$ \\
\hline Besoins psycho. famille & $\begin{array}{c}5,11 * \\
{[4,77} \\
5,57]\end{array}$ & $\begin{array}{c}5,32 * \\
{[4,62,} \\
6,41]\end{array}$ & $\begin{array}{c}5,26 * \\
{[4,66,} \\
6,28]\end{array}$ & $\begin{array}{c}5,15^{*} \\
{[4,48,} \\
6,47]\end{array}$ & $\begin{array}{c}4,54 * \\
{[3,51,} \\
6,02]\end{array}$ & $\begin{array}{c}3,95 * \\
{[2,62,} \\
7,15]\end{array}$ & $\begin{array}{c}4,22 * \\
{[3,51,} \\
5,81]\end{array}$ & $\begin{array}{c}4,39 * \\
{[3,58,} \\
6,02]\end{array}$ \\
\hline Besoins psycho. amis & $\begin{array}{c}3,17 * \\
{[2,91} \\
3,38]\end{array}$ & $\begin{array}{c}3,15^{*} \\
{[2,72} \\
3,81]\end{array}$ & $\begin{array}{c}3,15^{*} \\
{[2,62,} \\
3,61]\end{array}$ & $\begin{array}{c}2,87 * \\
{[2,49} \\
3,51]\end{array}$ & $\begin{array}{c}3,43^{*} \\
{[2,65} \\
4,52]\end{array}$ & $\begin{array}{c}2,86^{*} \\
{[2,46,} \\
5,01]\end{array}$ & $\begin{array}{c}4,39 * \\
{[3,51} \\
5,81]\end{array}$ & $\begin{array}{c}2,60 * \\
{[2,01} \\
3,48]\end{array}$ \\
\hline Besoins psycho. école & $\begin{array}{c}3,85^{*} \\
{[3,58} \\
4,18]\end{array}$ & $\begin{array}{c}4,06^{*} \\
{[3,52,} \\
4,53]\end{array}$ & $\begin{array}{c}3,88 * \\
{[3,45} \\
4,51]\end{array}$ & $\begin{array}{c}3,85 * \\
{[3,42,} \\
4,49]\end{array}$ & $\begin{array}{c}4,29 * \\
{[3,38,} \\
5,48]\end{array}$ & $\begin{array}{c}4,75^{*} \\
{[3,48,} \\
6,98]\end{array}$ & $\begin{array}{c}3,49 * \\
{[3,46,} \\
5,95]\end{array}$ & $\begin{array}{c}3,25^{*} \\
{[2,48,} \\
4,01]\end{array}$ \\
\hline Sécurité communautaire & $\begin{array}{c}1,84 * \\
{[1,74,} \\
1,96]\end{array}$ & $\begin{array}{c}1,87 * \\
{[1,69,} \\
2,15]\end{array}$ & $\begin{array}{c}1,82 * \\
{[1,62,} \\
2,08]\end{array}$ & $\begin{array}{c}2,00 * \\
{[1,78,} \\
2,36]\end{array}$ & $\begin{array}{c}1,69 * \\
{[1,48,} \\
2,23]\end{array}$ & $\begin{array}{c}1,52 * \\
{[1,28,} \\
2,38]\end{array}$ & $\begin{array}{c}1,52 * \\
{[1,28,} \\
1,82]\end{array}$ & $\begin{array}{c}2,00 * \\
{[1,68,} \\
2,52]\end{array}$ \\
\hline Insécurité alimentaire & $\begin{array}{c}0,57^{*} \\
{[0,53} \\
0,63]\end{array}$ & $\begin{array}{c}0,59 * \\
{[0,52,} \\
0,68]\end{array}$ & $\begin{array}{c}0,57^{*} \\
{[0,48} \\
0,66]\end{array}$ & $\begin{array}{c}0,55 * \\
{[0,48,} \\
0,68]\end{array}$ & $\begin{array}{c}0,55^{*} \\
{[0,48,} \\
0,75]\end{array}$ & $\begin{array}{c}0,64 * \\
{[0,45,} \\
0,98]\end{array}$ & $\begin{array}{c}0,59 * \\
{[0,49} \\
0,78]\end{array}$ & $\begin{array}{c}0,54 * \\
{[0,42,} \\
0,71]\end{array}$ \\
\hline Exercice & $\begin{array}{c}1,31 * \\
{[1,22,} \\
1,42]\end{array}$ & $\begin{array}{c}1,33 * \\
{[1,18} \\
1,61]\end{array}$ & $\begin{array}{c}1,16^{*} \\
{[1,05} \\
1,38]\end{array}$ & $\begin{array}{c}1,18 * \\
{[1,05} \\
1,42]\end{array}$ & $\begin{array}{c}1,51 * \\
{[1,11} \\
1,92]\end{array}$ & $\begin{array}{c}1,49 * \\
{[1,22} \\
2,44]\end{array}$ & $\begin{array}{c}1,48 * \\
{[1,21} \\
1,88]\end{array}$ & $\begin{array}{c}1,34 * \\
{[1,05} \\
1,75]\end{array}$ \\
\hline Tabac quoti. ou occasionnel & $\begin{array}{c}0,53 * \\
{[0,49} \\
0,57]\end{array}$ & $\begin{array}{c}0,49 * \\
{[0,43} \\
0,58]\end{array}$ & $\begin{array}{c}0,52 * \\
{[0,46} \\
0,63]\end{array}$ & $\begin{array}{c}0,47 * \\
{[0,43} \\
0,58]\end{array}$ & $\begin{array}{c}0,61 * \\
{[0,46,} \\
0,81]\end{array}$ & $\begin{array}{c}0,65 * \\
{[0,41} \\
0,95]\end{array}$ & $\begin{array}{c}0,56^{*} \\
{[0,43} \\
0,73] \\
\end{array}$ & $\begin{array}{c}0,62 * \\
{[0,46} \\
0,78]\end{array}$ \\
\hline Betas & NB & 1 & 2 & 3 & 4 & 5 & 6 & 7 \\
\hline Males & $-0,22$ & $-0,20$ & $-0,20$ & $-0,13$ & $-0,39$ & $-0,28$ & $-0,36$ & $-0,22$ \\
\hline Statut LGBTQ & $-0,56$ & $-0,60$ & $-0,61$ & $-0,51$ & $-0,45$ & $-0,50$ & $-0,65$ & $-0,26$ \\
\hline Besoins d'apprentissage & $-0,27$ & $-0,26$ & $-0,27$ & $-0,35$ & $-0,25$ & $-0,09$ & $-0,14$ & $-0,35$ \\
\hline Environnement bâti et naturel & 0,41 & 0,45 & 0,52 & 0,39 & 0,50 & 0,30 & 0,28 & 0,19 \\
\hline Besoins psycho. famille & 1,64 & 1,69 & 1,67 & 1,65 & 1,55 & 1,52 & 1,48 & 1,48 \\
\hline Besoins psycho. amis & 1,15 & 1,18 & 1,12 & 1,08 & 1,22 & 1,15 & 1,52 & 0,90 \\
\hline Besoins psycho. école & 1,36 & 1,39 & 1,37 & 1,34 & 1,42 & 1,58 & 1,30 & 1,15 \\
\hline Sécurité communautaire & 0,62 & 0,64 & 0,60 & 0,71 & 0,55 & 0,50 & 0,41 & 0,71 \\
\hline Insécurité alimentaire & $-0,54$ & $-0,52$ & $-0,57$ & $-0,56$ & $-0,55$ & $-0,42$ & $-0,50$ & $-0,62$ \\
\hline Exercice & 0,27 & 0,31 & 0,18 & 0,20 & 0,41 & 0,51 & 0,40 & 0,27 \\
\hline Tabac quoti. ou occasionnel & $-0,64$ & $-0,70$ & $-0,62$ & $-0,71$ & $-0,48$ & $-0,50$ & $-0,57$ & $-0,50$ \\
\hline
\end{tabular}

\section{Réussite scolaire}

Le Tableau 5 présente les résultats des régressions logistiques bayésiennes pour la réussite scolaire. Tout d'abord, le déterminant des besoins psychologiques comblés à 
l'école se démarque des autres déterminants dans sa capacité à prédire la réussite scolaire attendue. En fait, au niveau provincial, les jeunes qui rapportent se sentir à leur place à l'école, en sécurité et traités de façon juste par leurs enseignants sont 3,48 fois (I.C. $95 \%$ $=[3.22,3.75])$ plus susceptibles d'atteindre les standards provinciaux de réussite scolaire. Cependant, les francophones et ceux qui ont des besoins d'apprentissage spéciaux n'ont qu'environ 0,40 fois la probabilité d'atteindre ces standards comparativement à leurs pairs. Bien que l'explication des raisons entrainant une différence entre les groupes linguistiques soit en dehors des objectifs de cet article, ces résultats reflètent en partie les tendances présentées dans la mise en contexte, soit celles des plus petites proportions de jeunes francophones qui réussissent en mathématiques et en français. Finalement, les jeunes qui consomment des produits tabagiques n'ont que 0,46 fois (I.C. $95 \%=[.41$, .54]) la probabilité d'atteindre la réussite comparativement à leurs confrères et consœurs.

\section{Tableau 5}

Rapports de cotes, intervalles de crédibilité à $95 \%$ et bêtas pour la réussite scolaire attendue

\begin{tabular}{|c|c|c|c|c|c|c|c|c|}
\hline \multirow[b]{2}{*}{ Variable - sous-groupe } & \multicolumn{8}{|c|}{ Zone } \\
\hline & NB & 1 & 2 & 3 & 4 & 5 & 6 & 7 \\
\hline \multicolumn{9}{|l|}{ Rapport de cotes } \\
\hline Langue - Français & $\begin{array}{c}0,39 * \\
{[0,36} \\
0,46]\end{array}$ & $\begin{array}{c}0,42 * \\
{[0,36} \\
0,47]\end{array}$ & $\begin{array}{c}0,41 * \\
{[0,37} \\
0,46]\end{array}$ & $\begin{array}{c}0,41 * \\
{[0,35} \\
0,45]\end{array}$ & $\begin{array}{c}0,40^{*} \\
{[0,37,} \\
0,44]\end{array}$ & $\begin{array}{c}0,41 * \\
{[0,36,} \\
0,45]\end{array}$ & $\begin{array}{c}0,42 * \\
{[0,36,} \\
0,45]\end{array}$ & $\begin{array}{c}0,40 * \\
{[0,38} \\
0,45]\end{array}$ \\
\hline Genre non-binaire & $\begin{array}{c}1,12 \\
{[0,81} \\
1,59]\end{array}$ & $\begin{array}{c}1,01 \\
{[0,83} \\
1,49]\end{array}$ & $\begin{array}{c}1,23 \\
{[0,91} \\
1,56]\end{array}$ & $\begin{array}{c}0,99 \\
{[0,81} \\
1,35]\end{array}$ & $\begin{array}{c}1,18 \\
{[0,79} \\
1,71]\end{array}$ & $\begin{array}{c}1,18 \\
{[0,81,} \\
1,65]\end{array}$ & $\begin{array}{c}1,21 \\
{[0,78} \\
1,45]\end{array}$ & $\begin{array}{l}1,18 \\
{[0,95} \\
1,61]\end{array}$ \\
\hline Statut immigrant & $\begin{array}{c}1,38^{*} \\
{[1,12,} \\
1,96]\end{array}$ & $\begin{array}{c}1,45^{*} \\
{[1,05} \\
1,95]\end{array}$ & $\begin{array}{c}1,39 * \\
{[1,03,} \\
1,87]\end{array}$ & $\begin{array}{c}1,37^{*} \\
{[1,08,} \\
1,65]\end{array}$ & $\begin{array}{c}1,39 * \\
{[1,12,} \\
1,82]\end{array}$ & $\begin{array}{c}1,38 * \\
{[1,05,} \\
1,83]\end{array}$ & $\begin{array}{c}1,41 * \\
{[1,02,} \\
1,91]\end{array}$ & $\begin{array}{c}1,34^{*} \\
{[1,11,} \\
1,78]\end{array}$ \\
\hline Besoins d'apprentissage & $\begin{array}{c}0,38^{*} \\
{[0,34} \\
0,44]\end{array}$ & $\begin{array}{c}0,39 * \\
{[0,34} \\
0,43]\end{array}$ & $\begin{array}{c}0,37 * \\
{[0,33} \\
0,43]\end{array}$ & $\begin{array}{c}0,39 * \\
{[0,35} \\
0,43]\end{array}$ & $\begin{array}{c}0,37^{*} \\
{[0,34} \\
0,42]\end{array}$ & $\begin{array}{c}0,37 * \\
{[0,35} \\
0,41]\end{array}$ & $\begin{array}{c}0,39 * \\
{[0,34} \\
0,43]\end{array}$ & $\begin{array}{c}0,38 * \\
{[0,35} \\
0,41]\end{array}$ \\
\hline Environnement bâti et naturel & $\begin{array}{c}0,87^{*} \\
{[0,76,} \\
0,96]\end{array}$ & $\begin{array}{c}0,85^{*} \\
{[0,75,} \\
0,97]\end{array}$ & $\begin{array}{c}0,87^{*} \\
{[0,77,} \\
0,94]\end{array}$ & $\begin{array}{c}0,84^{*} \\
{[0,76} \\
0,95]\end{array}$ & $\begin{array}{c}0,86^{*} \\
{[0,76,} \\
0,98]\end{array}$ & $\begin{array}{c}0,86^{*} \\
{[0,75,} \\
0,94]\end{array}$ & $\begin{array}{c}0,83 * \\
{[0,77} \\
0,93]\end{array}$ & $\begin{array}{c}0,85^{*} \\
{[0,76} \\
0,94]\end{array}$ \\
\hline Besoins psycho. école & $\begin{array}{c}3,48 * \\
{[3,22,} \\
3,75]\end{array}$ & $\begin{array}{c}3,50 * \\
{[3,12} \\
3,85]\end{array}$ & $\begin{array}{c}3,42 * \\
{[3,07,} \\
3,81]\end{array}$ & $\begin{array}{c}3,41 * \\
{[3,11} \\
3,80]\end{array}$ & $\begin{array}{c}3,50 * \\
{[3,15,} \\
3,78]\end{array}$ & $\begin{array}{c}3,47 * \\
{[3,09} \\
3,80]\end{array}$ & $\begin{array}{c}3,38^{*} \\
{[3,05,} \\
3,75]\end{array}$ & $\begin{array}{c}3,49 * \\
{[3,12} \\
3,68]\end{array}$ \\
\hline Sécurité communautaire & $\begin{array}{c}1,17^{*} \\
{[1,08,} \\
1,26]\end{array}$ & $\begin{array}{c}1,18^{*} \\
{[1,07,} \\
1,28]\end{array}$ & $\begin{array}{c}1,16^{*} \\
{[1,06,} \\
1,24]\end{array}$ & $\begin{array}{c}1,14^{*} \\
{[1,07,} \\
1,23]\end{array}$ & $\begin{array}{c}1,15^{*} \\
{[1,07} \\
1,24]\end{array}$ & $\begin{array}{c}1,14 * \\
{[1,06} \\
1,23]\end{array}$ & $\begin{array}{c}1,16^{*} \\
{[1,07,} \\
1,24]\end{array}$ & $\begin{array}{c}1,14^{*} \\
{[1,06} \\
1,23]\end{array}$ \\
\hline Insécurité alimentaire & $\begin{array}{c}0,61 * \\
{[0,54} \\
0,66]\end{array}$ & $\begin{array}{c}0,60 * \\
{[0,56} \\
0,64]\end{array}$ & $\begin{array}{c}0,59 * \\
{[0,54} \\
0,64]\end{array}$ & $\begin{array}{c}0,58 * \\
{[0,55,} \\
0,64]\end{array}$ & $\begin{array}{c}0,62 * \\
{[0,54,} \\
0,64]\end{array}$ & $\begin{array}{c}0,60 * \\
{[0,55} \\
0,63]\end{array}$ & $\begin{array}{c}0,58^{*} \\
{[0,54} \\
0,63]\end{array}$ & $\begin{array}{c}0,59 * \\
{[0,56} \\
0,64]\end{array}$ \\
\hline Exercice & $\begin{array}{c}1,26^{*} \\
{[1,18} \\
1,42]\end{array}$ & $\begin{array}{c}1,34^{*} \\
{[1,15,} \\
1,58]\end{array}$ & $\begin{array}{c}1,24^{*} \\
{[1,11,} \\
1,48]\end{array}$ & $\begin{array}{c}1,35^{*} \\
{[1,12,} \\
1,49]\end{array}$ & $\begin{array}{c}1,28^{*} \\
{[1,11,} \\
1,43]\end{array}$ & $\begin{array}{c}1,30 * \\
{[1,12,} \\
1,45]\end{array}$ & $\begin{array}{c}1,28^{*} \\
{[1,13} \\
1,43]\end{array}$ & $\begin{array}{c}1,22^{*} \\
{[1,12} \\
1,42]\end{array}$ \\
\hline
\end{tabular}




\begin{tabular}{lcccccccc} 
Tabac quoti. ou occasionnel & $0,46^{*}$ & $0,48^{*}$ & $0,46^{*}$ & $0,44^{*}$ & $0,48^{*}$ & $0,49 *$ & $0,46^{*}$ & $0,45^{*}$ \\
& {$[0,41$,} & {$[0,40$,} & {$[0,38$,} & {$[0,41$,} & {$[0,42$,} & {$[0,42$,} & {$[0,40$,} & {$[0,39$,} \\
& $0,54]$ & $0,53]$ & $0,52]$ & $0,53]$ & $0,52]$ & $0,51]$ & $0,53]$ & $0,54]$ \\
Alcool mensuel & $0,83^{*}$ & $0,85^{*}$ & $0,81^{*}$ & $0,84^{*}$ & $0,85^{*}$ & $0,84^{*}$ & $0,82^{*}$ & $0,80^{*}$ \\
& {$[0,74$,} & {$[0,75$,} & {$[0,75$,} & {$[0,76$,} & {$[0,74$,} & {$[0,75$,} & {$[0,76$,} & {$[0,75$,} \\
& $0,92]$ & $0,91]$ & $0,92]$ & $0,93]$ & $0,91]$ & $0,89]$ & $0,91]$ & $0,89]$ \\
Risques de blessures & $0,75^{*}$ & $0,79 *$ & $0,75^{*}$ & $0,77^{*}$ & $0,74^{*}$ & $0,75^{*}$ & $0,73^{*}$ & $0,74^{*}$ \\
& {$[0,66$,} & {$[0,68$,} & {$[0,66$,} & {$[0,67$,} & {$[0,66$,} & {$[0,68$,} & {$[0,66$,} & {$[0,68$,} \\
& $0,86]$ & $0,88]$ & $0,84]$ & $0,85]$ & $0,83]$ & $0,84]$ & $0,85]$ & $0,83]$ \\
\hline Betas & $\mathrm{NB}$ & 1 & 2 & 3 & 4 & 5 & 6 & 7 \\
\hline Langue - Français & $-0,91$ & $-0,91$ & $-0,91$ & $-0,91$ & $-0,91$ & $-0,91$ & $-0,90$ & $-0,90$ \\
Genre non-binaire & 0,11 & 0,15 & 0,13 & 0,09 & 0,12 & 0,12 & 0,08 & 0,19 \\
Statut immigrant & 0,31 & 0,33 & 0,34 & 0,29 & 0,34 & 0,24 & 0,32 & 0,35 \\
Besoins d'apprentissage & $-0,94$ & $-0,95$ & $-0,96$ & $-0,95$ & $-0,96$ & $-0,95$ & $-0,94$ & $-0,96$ \\
Environnement bâti et naturel & $-0,15$ & $-0,16$ & $-0,17$ & $-0,16$ & $-0,16$ & $-0,17$ & $-0,16$ & $-0,16$ \\
Besoins psycho. école & 1,22 & 1,23 & 1,23 & 1,23 & 1,24 & 1,24 & 1,22 & 1,24 \\
Sécurité communautaire & 0,14 & 0,14 & 0,14 & 0,13 & 0,14 & 0,14 & 0,13 & 0,13 \\
Insécurité alimentaire & $-0,52$ & $-0,53$ & $-0,52$ & $-0,53$ & $-0,52$ & $-0,52$ & $-0,52$ & $-0,53$ \\
Exercice & 0,25 & 0,25 & 0,24 & 0,25 & 0,25 & 0,25 & 0,25 & 0,25 \\
Tabac quoti. ou occasionnel & $-0,78$ & $-0,78$ & $-0,78$ & $-0,79$ & $-0,77$ & $-0,75$ & $-0,78$ & $-0,78$ \\
Alcool mensuel & $-0,19$ & $-0,19$ & $-0,19$ & $-0,19$ & $-0,19$ & $-0,19$ & $-0,19$ & $-0,19$ \\
Risques de blessures & $-0,29$ & $-0,28$ & $-0,29$ & $-0,27$ & $-0,29$ & $-0,29$ & $-0,29$ & $-0,28$ \\
\hline
\end{tabular}

\section{Résultats des analyses de profils fictifs}

Afin de contraster l'effet des déterminants de la santé sur la résilience et la réussite scolaire entre les zones de santé du Nouveau-Brunswick, des profils d'élèves fictifs ont été créés. Ces profils utilisent certains déterminants pour illustrer comment un même élève peut être plus ou moins vulnérable selon la zone.

Résilience, profil A. Le profil A fait voir un jeune homme (présence du facteur de protection du sexe mâle) assez populaire avec ses pairs (présence du facteur de protection des besoins psychologiques comblés par les amis), mais en salle de classe, sa tendance à bavarder avec ses amis et ses besoins d'apprentissage spéciaux (présence du facteur de vulnérabilité des besoins d'apprentissages) ajoutent une charge de travail aux enseignantes, les rendant impatientes avec lui (absence du facteur de protection des besoins psychologiques comblés à l'école). De plus, étant fils d'une mère monoparentale très occupée (absence du facteur de protection des besoins psychologiques comblés par la famille), ce jeune homme souffre d'insécurité alimentaire (présence du facteur de vulnérabilité de l'insécurité alimentaire). Malgré ces défis, il a réussi à se tailler une place dans l'équipe de ballon-volant de son école secondaire (présence du facteur de protection de l'exercice). La Figure 2 présente les probabilités que cet élève ait un niveau de résilience élevé ou moyen selon la zone de santé. 


\section{Figure 2}

Probabilités de résilience, profil A

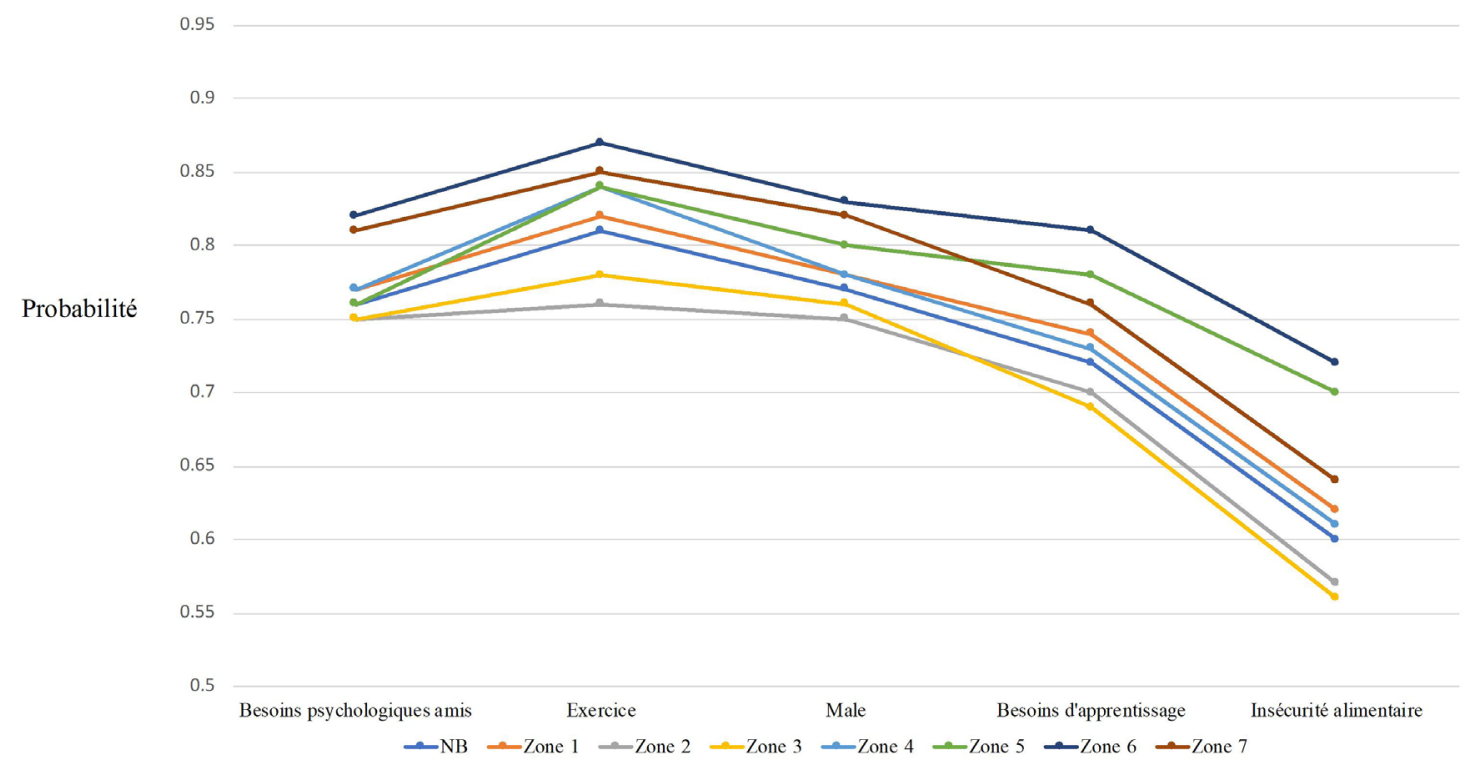

Les résultats illustrent que la probabilité qu'un élève avec ce profil ait un niveau élevé ou moyen de résilience diffère beaucoup d'une zone de santé à l'autre. Dans la zone de Bathurst/Péninsule acadienne (zone 6), ce jeune homme aurait une probabilité de résilience de $72 \%$, tandis que cette probabilité n'est que de $56 \%$ dans la zone de Fredericton/La vallée (zone 3). Cette figure démontre aussi la tendance protectrice de l'exercice, surtout dans la zone de santé 5 (Restigouche), où la probabilité de résilience augmente de $76 \%$ à $84 \%$ avec l'ajout de ce déterminant de santé. Finalement, l'effet dévastateur de l'insécurité alimentaire est démontré, réduisant les probabilités de résilience de $12 \%$ en moyenne.

Résilience, profil B. Pour le profil B, on trouve une adolescente (absence du facteur de protection du sexe mâle) homosexuelle (présence du facteur de vulnérabilité LGBTQ) qui consomme des produits tabagiques aux récréations (présence du facteur de vulnérabilité de l'utilisation du tabac). Bien qu'elle vive parfois de la discrimination de ses pairs (absence du facteur de protection des besoins psychologiques comblés par les amis) en raison de son orientation sexuelle, le personnel enseignant la fait se sentir à l'aise à l'école et soutient bien son apprentissage (présence du facteur de protection des besoins psychologiques comblés à l'école). Elle considère son quartier comme 
sécuritaire et peut se fier aux gens de son voisinage (présence du facteur de protection de la sécurité communautaire). De plus, quand elle se dispute avec ses parents à la maison, elle peut facilement trouver un endroit dans les environs où aller relaxer et reprendre ses forces (présence du facteur de protection de l'environnement bâti et naturel). La Figure 3 présente les probabilités de résilience de cette élève selon la zone de santé.

\section{Figure 3}

Probabilités de résilience, profil $B$

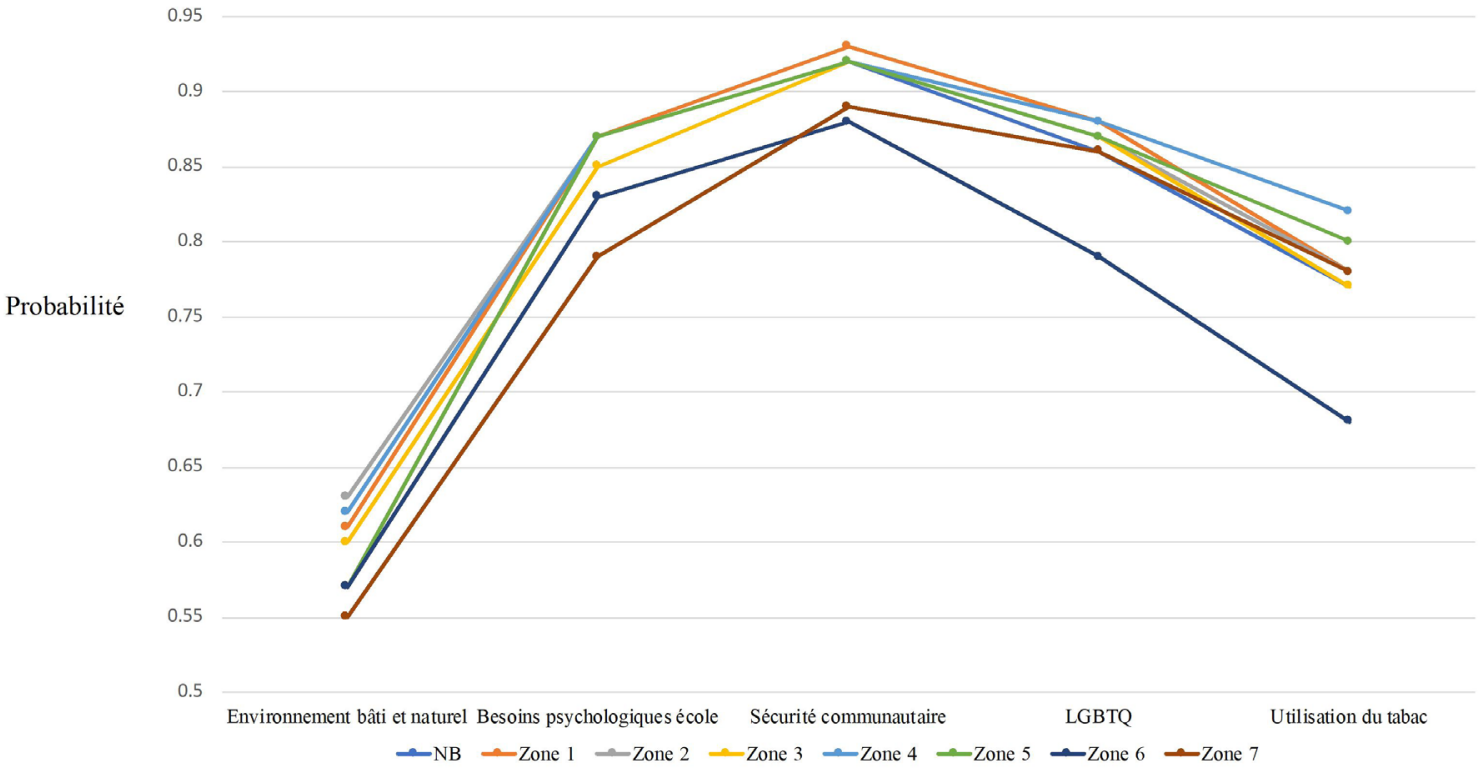

Bien que les probabilités de résilience suivent des parcours assez similaires pour ce profil, une zone semble faire exception, mettant la jeune fille décrite significativement plus à risque. Plus précisément, la probabilité de résilience dans la plupart des zones gravite autour de $80 \%$ pour ce profil; par contre, elle se situe seulement à $68 \%$ dans la zone 6, celle de Bathurst/Péninsule acadienne. Il est intéressant de noter que c'est en fait la même zone, celle de Bathurst/Péninsule acadienne, où le jeune homme décrit au profil A avait le plus de chance d'être résilient. Les résultats de la Figure 3 illustrent aussi l'effet protecteur des besoins psychologiques comblés à l'école, augmentant d'environ $25 \%$ les probabilités de résilience pour toutes les zones. Finalement, on observe une diminution importante de la probabilité de résilience dans la zone de Bathurst/Péninsule acadienne à l'ajout du déterminant du statut LGBTQ. 
Réussite scolaire, profil C. Dans le profil C, l'élève considère son genre comme étant fluide (présence du facteur du genre non-binaire). Le personnel enseignant respecte son identité et fait les accommodements nécessaires pour que l'élève se sente accepté à l'école (présence du facteur de protection des besoins psychologiques comblés à l'école). Malgré ces facteurs, l'élève souffre d'insécurité alimentaire (présence du facteur de vulnérabilité de l'insécurité alimentaire) et a tendance à fumer pour se détendre (présence du facteur de vulnérabilité de l'utilisation du tabac). De plus, l'élève adopte certains comportements à risque de provoquer des blessures graves (présence du facteur de vulnérabilité des risques de blessures). Par exemple, cette personne ne porte jamais sa ceinture de sécurité et ne traverse pas les rues aux endroits désignés. La Figure 4 présente les probabilités que l'élève ait une réussite scolaire moyenne ou au-delà de la moyenne.

\section{Figure 4}

Probabilités de réussite scolaire, profil C

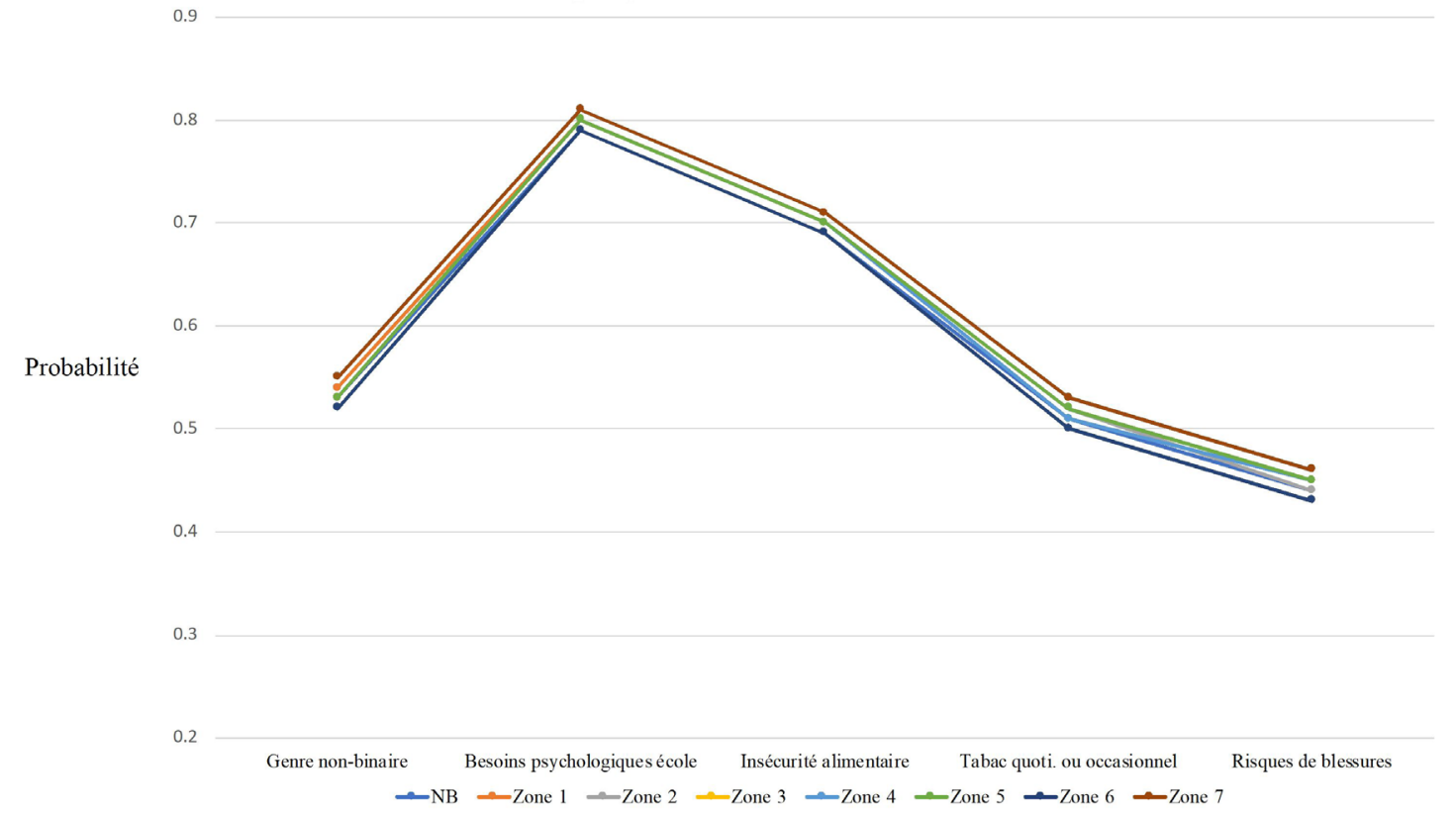

L'élève décrit au profil C aurait en moyenne $45 \%$ de chance de réussir à l'école. Entre les zones, les probabilités diffèrent très peu. En fait, à la suite de l'ajout du dernier déterminant, la zone offrant à l'étudiant la probabilité la plus élevée de réussite scolaire attendue est la zone 7 (Miramichi), où l'élève aurait $46 \%$ de chance, mais la zone offrant la probabilité de réussite la plus faible, Bathurst/Péninsule acadienne (zone 6), n'est 
que $3 \%$ plus faible, à $43 \%$. Par contre, cette figure illustre bien l'effet protecteur des besoins psychologiques comblés à l'école, ainsi que l'effet néfaste de la consommation de produits tabagiques sur la réussite scolaire au niveau attendu.

Réussite scolaire, profil D. Pour ce dernier profil, on trouve un nouvel arrivant francophone (présence du facteur de vulnérabilité de la langue) de la Côte d'Ivoire (présence du facteur de protection d'être immigrant). Comparativement aux circonstances difficiles dans son pays d'origine, il sent que sa communauté est très sécuritaire (présence du facteur de protection de la sécurité communautaire). En salle de classe, il a un peu de difficulté à suivre le rythme des apprentissages, donc il nécessite une adaptation scolaire (présence du facteur de vulnérabilité des besoins d'apprentissage). Cependant, ses habiletés sportives font de lui un élève modèle au cours d'éducation physique. Son enseignant l'invite donc à entrainer les plus jeunes au soccer après l'école, une offre qu'il accepte avec fierté (présence du facteur de protection de l'exercice). La Figure 5 présente les probabilités pour cet élève d'atteindre le seuil de réussite scolaire attendu.

\section{Figure 5}

Probabilités de réussite scolaire, profil D

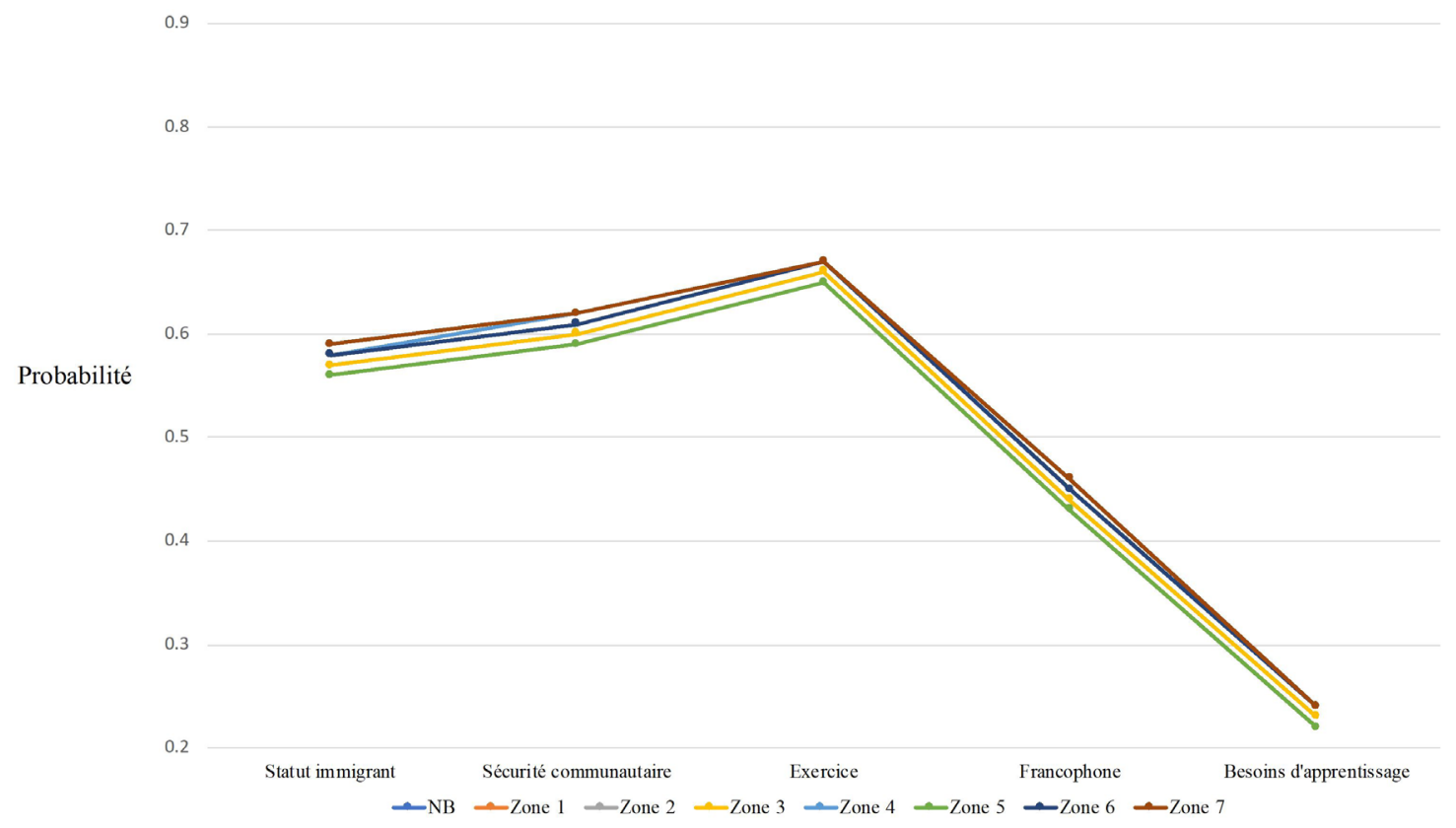


Malgré la présence de trois déterminants de protection, ses besoins d'apprentissage font en sorte que sa probabilité de réussite scolaire n'est que d'environ $23 \%$. Entre les zones, les probabilités de réussite scolaire attendue diffèrent très peu, ne dépassant pas une différence de probabilité de $5 \%$, et en considérant l'erreur d'échantillonnage inévitable, celles-ci risquent d'être identiques. Par contre, il est intéressant de noter la baisse importante de la probabilité de réussite scolaire attendue dans toutes les zones à la suite de l'ajout du déterminant linguistique. L'élaboration des facteurs explicatifs de cet effet dépasse l'objectif de l'article, mais il est certainement possible qu'une partie de la variance expliquée par le déterminant linguistique soit liée à d'autres déterminants du modèle, par exemple à des différences dans les pratiques de consommation ou sur le plan de l'insécurité alimentaire. Il est aussi impossible d'exclure les différences concernant l'évaluation de la réussite entre les districts scolaires francophones et anglophones en tant que causes possibles.

\section{Discussion}

Les objectifs de cette étude étaient 1) d'identifier les déterminants de la santé les plus importants pour la résilience et la réussite scolaire chez les jeunes de 12 à 18 ans du Nouveau-Brunswick et 2) d'en contraster l'effet dans les 7 zones de santé de la province. Des régressions logistiques fréquentielles ont permis de faire un tri préliminaire des déterminants, et des régressions logistiques bayésiennes ont permis d'intégrer des connaissances antérieures sur les effets de ces déterminants sur la résilience et la réussite scolaire, ainsi que de préciser l'importance de ces effets. En fait, on remarque que les intervalles de crédibilité des résultats bayésiens sont, pour la majorité, plus restreints que ceux des résultats fréquentiels, se traduisant en des estimations à postériori plus précises. À la suite des régressions logistiques bayésiennes, des profils fictifs d'étudiants ont été créés afin de contraster l'effet des déterminants sur la vulnérabilité d'un même jeune entre les sept zones de santé du Nouveau-Brunswick. Cette utilisation innovatrice des estimations bayésiennes a permis d'illustrer les différences entre les zones et de comparer les différents effets d'un même déterminant entre les zones.

Pour ce qui est des résultats de l'étude, la satisfaction des besoins psychologiques par la famille, les amis et à l'école est le déterminant de la santé qui prédit le plus 
fortement la résilience. En fait, les résultats indiquent que les élèves de la province qui rapportent se sentir soutenus psychologiquement par leur famille, leurs amis, ou à l'école sont de 3,38 à 5,11 fois plus susceptibles d'avoir une résilience moyenne ou élevée. Ces résultats corroborent les conclusions d'études précédentes. En fait, Zolkoski et Bullock (2012) ont recensé les articles de 1950 à 2010 portant sur les déterminants de la résilience et ont soulevé que les conditions familiales et le soutien de la communauté englobaient la majorité des effets protecteurs de la résilience. Plus précisément, les parents qui créent un environnement stable, stimulant et affectueux à la maison tendent à avoir des enfants plus résilients. De plus, Zolkoski et Bullock (2012) ont avancé que les environnements accueillants créés par les gens de la communauté du jeune, comme les enseignants, les psychologues scolaires et les entraineurs, agissent comme des tremplins pour promouvoir la résilience chez les jeunes à risque.

Les jeunes de la communauté LGBTQ semblent plus à risque d'une résilience faible que leurs pairs hétérosexuels. Par contre, Russell (2005) et Russell et Fish (2016) laissent entendre que la différence de résilience entre les jeunes hétérosexuels et homosexuels ne se trouve pas dans la quantité de déterminants prédisant la résilience, mais plutôt dans l'intensité de ces déterminants. Par exemple, un environnement accueillant où l'on se sent accepté et valorisé est important pour tous, peu importe l'orientation sexuelle, mais chez les jeunes homosexuels, l'absence d'un tel environnement pourrait dériver de la victimisation, de l'homophobie et de l'intimidation. De plus, Russell (2005) fait remarquer que l'orientation sexuelle a des répercussions sur plusieurs niveaux contextuels. Plus précisément, l'acceptation ou le rejet de l'homosexualité peuvent être présents tant sur le plan psychologique de l'individu que sur la plan politique, créant des barrières supplémentaires au développement de la résilience.

Un déterminant qui s'est montré significatif tant pour la résilience que pour la réussite scolaire est la consommation de produits tabagiques. Bien que l'effet n'en soit pas un de causalité, mais plutôt de corrélation, les probabilités de résilience et de réussite scolaire sont réduites de moitié lorsque l'élève consomme des produits tabagiques. Pour la résilience, ces résultats sont très peu surprenants, car lorsque Reid et al. (2017) ont demandé aux Canadiens d'autoévaluer leur santé mentale, 77,4 \% des non-fumeurs ont déclaré avoir une excellente ou une très bonne santé mentale, alors que ce n'était le cas que chez $65,0 \%$ des fumeurs, reflétant possiblement un taux plus faible de résilience aux exigences de la vie. En ce qui concerne les effets de la consommation de produits 
tabagiques sur la réussite scolaire, les résultats des régressions logistiques bayésiennes effectuées dans cette étude viennent soutenir les conclusions de Bradley et Greene (2013), qui rapportent que les 28 articles de leur recension des écrits sur le sujet ont trouvé des associations inversement significatives entre la consommation de produits tabagiques et la réussite scolaire.

Tout comme pour la résilience, les besoins psychologiques satisfaits à l'école constituent un facteur de protection significatif pour la réussite scolaire. Les jeunes qui se sentent écoutés, respectés et accueillis dans leur milieu scolaire sont 3,48 fois plus susceptibles d'avoir un rendement scolaire moyen ou au-delà de la moyenne. En fait, Wang et Holcombe (2010) parviennent à la conclusion que la perception des élèves de leur environnement scolaire influence la réussite scolaire en augmentant leur participation en classe, leur sentiment d'appartenance et leur utilisation de stratégies d'autorégulation lors de l'étude. Quelques années plus tard, Wang et Eccles (2013) et King (2015) ont également appuyé ces associations. Par contre, King (2015) arrive à la conclusion que les besoins psychologiques comblés par les parents de l'élève seraient plus importants pour la réussite scolaire que la satisfaction de ces besoins à l'école, ce qui n'a pas été démontré dans notre étude. Des recherches supplémentaires sur ce sujet sont donc nécessaires.

Finalement, les élèves avec des programmes d'apprentissage spéciaux n'ont que 0,38 fois les chances de réussir à l'école comparativement à leurs pairs. En fait, ce déterminant est le plus puissant dans sa capacité à prédire une faible réussite scolaire dans toutes les zones de la province. Ce résultat était attendu, car plusieurs études sur le sujet ont rapporté des résultats similaires. Entre autres, les résultats des études de Forrest et al. (2011) et de Daley et Birchwood (2010) corroborent les résultats de cette étude.

Pour ce qui est des résultats des analyses de profils, les deux profils créés pour la résilience ont démontré que la zone 6, la zone de Bathurst/Péninsule acadienne, est la zone mettant le jeune du profil A le moins à risque d'une faible résilience, mais la jeune du profil $\mathrm{B}$ le plus à risque; ce résultat étant largement dû aux effets moins prononcés des besoins d'apprentissages et de l'insécurité alimentaire dans le profil A, et à l'effet moins prononcé de la sécurité communautaire dans le profil B. De plus, les comparaisons pour la résilience ont fait ressortir l'efficacité de l'exercice physique comme facteur de protection dans la zone 5, celle de Restigouche, ainsi que l'augmentation du risque pour les jeunes de la communauté LGBTQ et pour ceux qui consomment des produits tabagiques dans la zone de Bathurst/Péninsule acadienne. Ces résultats obtenus à l'aide 
des profils fictifs pourront servir à mieux orienter les efforts d'intervention, car ils illustrent l'importance de l'effet de chaque déterminant selon les zones. Conséquemment, ces profils permettent de voir que l'amélioration d'un certain déterminant, comme la sécurité communautaire, n'aura pas un effet uniforme sur la résilience dans toutes les zones. Cette analyse selon nous novatrice pourrait donc avoir des répercussions intéressantes sur le plan pratique.

\section{Conclusion}

Cette étude a permis d'illustrer les particularités des zones de santé du NouveauBrunswick quant à leur probabilité de promouvoir la résilience et la réussite scolaire. La satisfaction des besoins psychologiques, l'insécurité alimentaire et la consommation de produits tabagiques ressortent comme des déterminants de la résilience et de la réussite scolaire. D'un point de vue statistique, cette étude a aussi permis d'illustrer l'intégration de méthodes bayésiennes en sciences sociales en démontrant son utilité et sa polyvalence tant pour la mise à jour des intervalles de crédibilité que pour les simulations de profils fictifs. L'inférence bayésienne a permis d'intégrer des lois à priori de sorte que les lois à postériori reflètent davantage l'état des connaissances.

Concernant les limites de l'étude, bien que l'analyse des profils fictifs ait permis de bien illustrer le changement de probabilité à la suite de l'ajout de chaque déterminant pour la réussite scolaire, elle n'a pas bien réussi à identifier des effets uniques aux zones. En d'autres mots, les probabilités demeurent très similaires entre les zones à la suite de l'ajout de chaque déterminant, ce qui n'est pas le cas pour les analyses en lien avec la résilience. Une explication possible pour ce résultat est que le modèle épidémiologique utilisé pour cette étude prend ses racines dans le domaine de la santé et non en pédagogie. Il est donc fort probable que d'autres déterminants, comme des mesures sur les besoins de santé, le harcèlement, la victimisation, l'engagement scolaire et la sécurité scolaire perçue par l'élève, doivent être ajoutés au modèle pour en affiner l'analyse (Bradley et Greene, 2013; Forrest et al., 2011; Milam et al., 2010; Wang et Eccles, 2013). L'ajout de ces déterminants permettrait sans doute d'améliorer la capacité du modèle à mieux déceler les jeunes ayant une forte probabilité de réussite de ceux ayant des probabilités plus faibles. 
De plus, les études basées sur des enquêtes sont limitées par leur devis corrélationnel, fournissant des preuves particulièrement faibles en ce qui concerne les relations de cause à effet. Par contre, les enquêtes avec un nombre aussi important de participants, un taux de réponse élevé et une méthode d'échantillonnage rigoureuse réduisent la probabilité d'avoir un échantillon non représentatif de la population. Par ailleurs, les auteurs ont consacré de nombreux mois à la lecture d'articles pour établir les à priori, mais les possibilités de les modifier légèrement en fonction d'articles nouvellement découverts sont infinies, ce qui oblige les chercheurs à décider quand cesser de recenser de nouveaux articles. Finalement, lorsque les articles recensés ne précisent pas de rapports de cotes ou d'intervalles de crédibilité précis pour les effets testés, les chercheurs doivent faire preuve de jugement quant à la valeur empirique des résultats.

Les études futures voulant identifier les déterminants de la réussite scolaire attendue devraient intégrer des déterminants d'ordres pédagogique et scolaire, ce que les enquêtes utilisées ici n'ont pas permis de faire. Cela dit, cette étude identifie quelques pistes d'action possibles pour l'amélioration de la réussite scolaire, notamment en traitant de l'insécurité alimentaire et en misant sur la satisfaction des besoins psychologiques dans le milieu scolaire. Finalement, il serait pertinent de reproduire cette étude avec un nouvel échantillon en utilisant les résultats de cette étude comme données à priori. Avec un système scolaire en évolution perpétuelle, cette reproduction permettrait de mettre à jour les effets de ces déterminants sur la résilience et la réussite scolaire. 


\section{Références}

American Psychological Association [APA]. (2017). Education and socioeconomic status. https://www.apa.org/pi/ses/resources/publications/factsheet-education.pdf

Balsa, A. I., Giuliano, L. M. et French, M. T. (2011). The effects of alcohol use on academic achievement in high school. Economics and Education Review, 30(1), 1-15. https://doi.org/10.1016/j.econedurev.2010.06.015

Barry, M. M. (2009). Addressing the determinants of positive mental health: concepts, evidence and practice. Journal of Mental Health Promotion, 11(3), 4-17. https:// doi.org/10.1080/14623730.2009.9721788

Berman, M. G., Jonides, J. et Kaplan, S. (2008). The cognitive benefits of interacting with nature. Psychological Science, 19(12), 1207-1212. https://doi. org/10.1111/j.1467-9280.2008.02225.x

Bolstad, W. M. et Curran, J. M. (2016). Introduction to Bayesian statistics (3e éd.). Wiley.

Booske, B. C., Athens, J. K., Kindig, D. A., Park, H. et Remington, P. L. (2010, février). Different perspectives for assigning weights to determinants of health [County health rankings working paper]. Population Health Institute. https://www.countyhealthrankings.org/sites/default/files/ differentPerspectivesForAssigningWeightsToDeterminantsOfHealth.pdf

Borofsky, L. A., Kellerman, I., Baucom, B., Oliver, P. H. et Margolin, G. (2013).

Community violence exposure and adolescents' school engagement and academic achievement over time. Psychology of violence, 3(4), 381-395. https://doi. org/10.1037/a0034121

Bourque, J. et El Adlouni, S.-E. (2016). Manuel d'introduction à la statistique appliquée aux sciences sociales. Presses de l'Université Laval.

Bourque, J., Doucet, D., LeBlanc, J., Dupuis, J. B. et Nadeau, J. (2019). L'alpha de Cronbach est l'un des pires estimateurs de la consistance interne : une étude de simulation. Revue des sciences de l'éducation, 45(2), 78-99. https://doi. org/10.7202/1067534ar

Bradley, B. J. et Greene, A. C. (2013). Do health and education agencies in the United States share responsibility for academic achievement and health? A review of 25 
years of evidence about the relationship of adolescents' academic achievement and health behaviors. Journal of Adolescent Health, 52(5), 523-532. https://doi. org/10.1016/j.jadohealth.2013.01.008

Cady, C. L. (2014). Food insecurity as a student issue. Journal of College and Character, 15(4), 265-272. https://doi.org/10.1515/jcc-2014-0031

Cohen, J. (1988). Statistical power analysis for the behavioral sciences (2e éd.). Lawrence Erlbaum.

Conseil de la santé du Nouveau-Brunswick [CSNB]. (2014). Déterminants de la santé [Modèle]. https://csnb.ca/le-modele-de-sante-de-la-population

Conseil de la santé du Nouveau-Brunswick [CSNB]. (2015). Favoriser la résilience dans les écoles et les collectivités du Nouveau-Brunswick. https://csnb.ca/sites/default/ files/publications-attachments/favoriser-la-resilience-dans-les-ecoles-et-lescollectivites-nb-2016011.pdf

Conseil de la santé du Nouveau-Brunswick [CSNB]. (2018). Sondage sur le mieux-être des élèves du Nouveau-Brunswick. https://csnb.ca/ sondage-sur-le-mieux-etre-des-eleves-du-nouveau-brunswick

Conseil de la santé du Nouveau-Brunswick [CSNB]. (2021). Cartes des zones de santé et des communautés $d u$ CSNB. https://csnb.ca/ cartes-des-zones-de-sante-et-des-communautes-du-csnb

Daley, D. et Birchwood, J. (2010). ADHD and academic performance: Why does ADHD impact on academic performance and what can be done to support ADHD children in the classroom? Child: Care, Health and Development, 36(4), 455-464. https://doi.org/10.1111/j.1365-2214.2009.01046.x

Deci, E. L. et Ryan, R. M. (2000). The "what" and "why" of goal pursuits: Human needs and the self-determination of behavior. Psychological Inquiry, 11(4), 227-268. https://doi.org/10.1207/S15327965PLI1104_01

Dezetter, A., Beaton, A. M. et Bourque, J. (2016, 2-3 novembre). Portrait of the mental health and use of care of youth in New Brunswick. Communication présentée à la 8 e conférence de la Fondation de la recherche en santé au Nouveau-Brunswick, Saint John, NB. 
Donnelly, J. E., Hillman, C. H., Castelli, D., Etnier, J. L., Lee, S., Tomporowski, P., Lambourne, K. et Szabo-Reed, A. N. (2016). Physical activity, fitness, cognitive function, and academic achievement in children: A systematic review. Medicine \& Science in Sports \& Exercise, 48(6), 1197-1222. https://doi.org/10.1249/ $\underline{\text { mss.0000000000000901 }}$

Faul, F., Erdfelder, E., Buchner, A. et Lang, A. G. (2009). Statistical power analyses using $\mathrm{G}^{*}$ Power 3.1: Tests for correlation and regression analyses. Behavior Research Methods, 41(4), 1149-1160. https://doi.org/10.3758/BRM.41.4.1149

Forrest, C. B., Bevans, K. B., Riley, A. W., Crespo, R. et Louis, T. A. (2011). School outcomes of children with special health care needs. Pediatrics, 128(2), 303-312. https://doi.org/10.1542/peds.2010-3347

Hair, E. C., Park, M. J., Ling, T. J. et Moore, K. A. (2009). Risky behaviors in late adolescence: Co-occurrence, predictors, and consequences. Journal of Adolescent Health, 45(3), 253-261. https://doi.org/10.1016/j.jadohealth.2009.02.009

Halpern, D. (2014). Mental health and the built environment: More than bricks and mortar? Routledge.

Herman, K. M., Hopman, W. M. et Sabiston, C. M. (2015). Physical activity, screen time, and self-rated health and mental health in Canadian adolescents. Preventive Medicine, 73, 112-116. https://doi.org/10.1016/j.ypmed.2015.01.030

IBM Corp. (2019). IBM SPSS Statistics for Windows (version 26.0) [Logiciel]. IBM Corp.

Institut canadien d'information sur la santé [ICIS]. (2020). Les soins aux enfants et aux jeunes atteints de troubles mentaux, 2020 - tableaux de données. https://www. cihi.ca/sites/default/files/document/children-youth-mental-health-data-tables-fr. $\underline{\mathrm{xlsx}}$

King, R. B. (2015). Sense of relatedness boosts engagement, achievement, and wellbeing: A latent growth model study. Contemporary Educational Psychology, 42, 26-38. https://doi.org/10.1016/j.cedpsych.2015.04.002

Little, R. J. A. et Rubin, D. B. (2002). Statistical analysis with missing data (2e éd.). John Wiley \& Sons. https://doi.org/10.1002/9781119013563 
Luthar, S. S. et Cicchetti, D. (2000). The construct of resilience: Implications for interventions and social policies. Development and Psychopathology, 12(4), 857-885. https://doi.org/10.1017/s0954579400004156

Masten, A. S., Hubbard, J. J., Gest, S. D., Tellegen, A., Garmezy, N. et Ramirez, M. (1999). Competence in the context of adversity: pathways to resilience and maladaptation from childhood to late adolescence. Development and psychopathology, 11(1), 143-169. https://doi.org/10.1017/s0954579499001996

Matlab. (2019). Version 9.6 (R2019a) [Logiciel]. The MathWorks Inc.

Melchior, M., Chastang, J.-F., Falissard, B., Galéra, C., Tremblay, R. E., Côté, S. M. et Boivin, M. (2012). Food insecurity and children's mental health: A prospective birth cohort study. PLoS ONE, 7(12), article e52615, 1-7. https://doi.org/10.1371/ journal.pone.0052615

Milam, A. J., Furr-Holden, C. D. M. et Leaf, P. J. (2010). Perceived school and neighborhood safety, neighborhood violence and academic achievement in urban school children. The Urban Review, 42(5), 458-467. https://dx.doi.org/10.1007/ s11256-010-0165-7

Ministère de l'Éducation et du Développement de la petite enfance du NouveauBrunswick [MEDPE-NB]. (2019a). Éducation et développement de la petite enfance : Rapport annuel 2018-2019. https://www2.gnb.ca/content/dam/gnb/ Departments/ed/pdf/Publications/RapportAnnuel2018-2019.pdf

Ministère de l'Éducation et du Développement de la petite enfance du NouveauBrunswick [MEDPE-NB]. (2019). Le bulletin provincial 2018-2019 : NouveauBrunswick francophone. https:/www2.gnb.ca/content/dam/gnb/Departments/ed/ pdf/K12/bulletin/2019/BULLETINNB.PDF

Ministère de l'Éducation et du Développement de la petite enfance du NouveauBrunswick [MEDPE-NB]. (2020). Provincial reports. https://www2.gnb.ca/ content/gnb/en/departments/education/k12/content/anglophone sector/reports on_achievement/provincial.html

Reading, C. et Wien, F. (2009). Health inequalities and social determinants of Aboriginal peoples' health. Centre de la collaboration nationale de la santé autochtone. 
https://www.ccnsa-nccah.ca/docs/determinants/RPT-HealthInequalities-ReadingWien-EN.pdf

Reid, J. L., Hammond, D., Rynard, V. L., Madill, C. L. et Burkhalter, R. (2017). Tobacco use in Canada: Patterns and trends, 2017 edition. Propel Centre for Population Health Impact, University of Waterloo. https://uwaterloo.ca/tobacco-use-canada/ sites/ca.tobacco-use-canada/files/uploads/files/2017_tobaccouseincanada_final _ 0. pdf

Robert, C. P. (2006). Le choix bayésien : principes et pratique. Springer.

Roustit, C., Hamelin, A.-M., Grillo, F., Martin, J. et Chauvin, P. (2010). Food insecurity: could school food supplementation help break cycles of intergenerational transmission of social inequalities? Pediatrics, 126(6), 1174-1181. https://doi. org/10.1542/peds.2009-3574

Russell, S. T. (2005). Beyond risk: Resilience in the lives of sexual minority youth. Journal of Gay \& Lesbian Issues in Education, 2(3), 5-18. https://doi. org $/ 10.1300 / \mathrm{J} 367 \mathrm{v} 02 \mathrm{n} 03 \quad 02$

Russell, S. T. et Fish, J. N. (2016). Mental health in lesbian, gay, bisexual, and transgender (LGBT) youth. Annual Review of Clinical Psychology, 12, 465-487. https://doi.org/10.1146/annurev-clinpsy-021815-093153

Sektnan, M., McClelland, M. M., Acock, A. et Morrison, F. J. (2010). Relations between early family risk, children's behavioral regulation, and academic achievement. Early Childhood Research Quarterly, 25(4), 464-479. https://doi.org/10.1016/j. ecresq.2010.02.005

Ungar, M. et Theron, L. (2020). Resilience and mental health: how multisystemic processes contribute to positive outcomes. The Lancet Psychiatry, 7(5), 441-448. https://doi.org/10.1016/s2215-0366(19)30434-1

Ungar, M., Liebenberg, L., Dudding, P., Armstrong, M. et van de Vijver, F. J. R. (2013). Patterns of service use, individual and contextual risk factors, and resilience among adolescents using multiple psychosocial services. Child Abuse and Neglect, 37(2-3), 150-159. https://doi.org/10.1016/j.chiabu.2012.05.007

Vable, A. M., Cohen, A. K., Leonard, S. A., Glymour, M. M., Duarte, C. D. P. et Yen, I. H. (2018). Do the health benefits of education vary by sociodemographic 
subgroup? Differential returns to education and implications for health inequities. Annals of Epidemiology, 28(11), 759-766.e5. https://doi.org/10.1016/j. annepidem.2018.08.014

van de Schoot, R., Kaplan, D., Denissen, J., Asendorpf, J. B., Neyer, F. J. et van Aken, M. A. G. (2014). A gentle introduction to Bayesian analysis: Applications to developmental research. Child Development, 85(3), 842-860. https://doi. org/10.1111/cdev.12169

Vanpaemel, W. et Lee, M. D. (2012). Using priors to formalize theory: Optimal attention and the generalized context model. Psychonomic Bulletin \& Review, 19(6), 1047-1056. https://doi.org/10.3758/s13423-012-0300-4

Wagenmakers, E.-J., Marsman, M., Jamil, T., Ly, A., Verhagen, J., Love, J., Selker, R., Gronau, Q. F., Šmíra, M., Epskamp, S., Matzke, D., Rouder, J. N. et Morey, R. D. (2018). Bayesian inference for psychology. Part I: Theoretical advantages and practical ramifications. Psychonomic Bulletin \& Review, 25(1), 35-57. https://doi. org/10.3758/s13423-017-1343-3

Wang, M.-T. et Eccles, J. S. (2013). School context, achievement motivation, and academic engagement: A longitudinal study of school engagement using a multidimensional perspective. Learning and Instruction, 28(1), 12-23. https://doi. org/10.1016/j.learninstruc.2013.04.002

Wang, M.-T. et Holcombe, R. (2010). Adolescents' perceptions of school environment, engagement, and academic achievement in middle school. American Educational Research Journal, 47(3), 633-662. https://doi.org/10.3102/0002831209361209

Wight, R. G., Botticello, A. L. et Aneshensel, C. S. (2006). Socioeconomic context, social support, and adolescent mental health: a multilevel investigation. Journal of Youth and Adolescence, 35(1), article 109, 115-126. https://doi.org/10.1007/ S10964-005-9009-2

Zolkoski, S. M. et Bullock, L. M. (2012). Resilience in children and youth: A review. Children and Youth Services Review, 34(12), 2295-2303. https://doi. org/10.1016/j.childyouth.2012.08.009 